\title{
Maps between local Picard groups
}

\author{
János Kollár
}

\begin{abstract}
The paper investigates maps between local Picard groups. The main goal is to understand the kernel in two cases: pull-back to the normalization and restriction to a hyperplane section. We give sufficient (and almost necessary) conditions that guarantee that the kernel is finite or of finite type.
\end{abstract}

\section{Introduction}

Let $X$ be a scheme and $x \in X$ a point. The local Picard group of $X$ at $x$, denoted by $\operatorname{Pic}^{\text {loc }}(x, X)$, is the Picard group of the punctured neighborhood $\operatorname{Spec}_{X} \mathcal{O}_{x, X} \backslash\{x\}$. Our aim is to study the pull-back map on the local Picard group in two situations.

Question 1.1 (Normalization). Let $\pi: \bar{X} \rightarrow X$ denote the normalization, and let $\bar{x}_{i} \in \bar{X}$ be the preimages of $x$. What is the kernel of the pull-back map

$$
\pi^{*}: \operatorname{Pic}^{\mathrm{loc}}(x, X) \rightarrow \sum_{i} \operatorname{Pic}^{\mathrm{loc}}\left(\bar{x}_{i}, \bar{X}\right) ?
$$

Question 1.2 (Restriction to a divisor). Let $x \in D \subset X$ be an effective Cartier divisor. What is the kernel of the restriction map

$$
r_{D}^{X}: \operatorname{Pic}^{\text {loc }}(x, X) \rightarrow \operatorname{Pic}^{\text {loc }}(x, D) ?
$$

In both cases we are interested in conditions that guarantee that the kernels of these maps are "small". Here "small" can mean trivial, or finite, or "naturally" a subgroup of a linear algebraic group.

Grothendieck's local Lefschetz-type theorem [Gro68, XI.3.16] is the first major result on Question 1.2. He proves that $r_{D}^{X}$ is injective if $\operatorname{depth}_{x} X \geqslant 4$. Conjecture 1.2 in [Kol13a] asserts that this can be relaxed to $\operatorname{depth}_{x} X \geqslant 3$ and $\operatorname{dim} X \geqslant 4$. This was proved in [Kol13a] when $X$ is $\log$ canonical and in [BdJ14] when $X$ is normal.

I was led to Question 1.1 while investigating stable varieties and their moduli. The method of [Kol13b] first studies the normalization $\bar{X}$ of a stable variety $X$ and then descends the information from $\bar{X}$ to $X$. It is especially important to know when the canonical divisor of $X$ is $\mathbb{Q}$-Cartier; this was settled in [Kol11]. For semi-log canonical varieties Question 1.1 is answered in [Kol13b, Section 5.7].

Received 2 September 2014, accepted in final form 3 January 2016.

2010 Mathematics Subject Classification 14J17, 14C22, 14F45, 32S30, 32S50.

Keywords: local Picard group, Grothendieck-Lefschetz hyperplane theorem, normalization.

This journal is (C) Foundation Compositio Mathematica 2016. This article is distributed with Open Access under the terms of the Creative Commons Attribution Non-Commercial License, which permits non-commercial reuse, distribution, and reproduction in any medium, provided that the original work is properly cited. For commercial re-use, please contact the Foundation Compositio Mathematica.

Partial financial support was provided by the NSF under grant numbers DMS-0968337 and DMS-1362960. 


\section{J. Kollár}

In many applications, the natural assumption is that $X$ satisfies Serre's condition $S_{2}$ only. To understand this case, one needs to know that, as in the global situation, the local Picard group is not just a group but a group scheme. The local definition is more involved than the projective one, and the natural setting turns out to be the following.

Condition 1.3. We consider a scheme $X$ that is essentially of finite type over a field $k$, and a 0-dimensional closed subscheme $x \subset X$. The scheme $X$ satisfies Serre's condition $S_{2}$ and has pure dimension at least 3 (that is, every associated prime of $\mathcal{O}_{X}$ has the same dimension, which is at least 3 ).

Boutot [Bou78] defines a local Picard functor Pic ${ }^{\text {loc }}(x, X)$ and proves that, under the above assumptions, it is represented by the local Picard scheme Pic ${ }^{\text {loc }}(x, X)$; see Definition 3.3 and [Bou78, Theorem 1.ii] for details. Frequently, $\mathbf{P i c}^{\text {loc }}(x, X)$ has infinitely many connected components. The identity component, usually denoted by $\mathbf{P i c}^{\text {loc-o}}(x, X)$, is a commutative, connected algebraic group of finite type, but it need not be a linear algebraic group. The union of those components of $\mathbf{P i c}{ }^{\mathrm{loc}}(x, X)$ that become torsion modulo $\mathbf{P i c}{ }^{\text {loc-o }}(x, X)$ is denoted by $\mathbf{P i c}{ }^{\text {loc }-\tau}(x, X)$.

The following theorem gives a unified answer to both questions.

Theorem 1.4. Let $X$ and $Y$ be schemes that satisfy Condition 1.3 and $\pi: Y \rightarrow X$ a finite morphism. Let $x \subset \pi(Y) \subset X$ be a 0-dimensional closed subscheme, $y \subset Y$ the preimage of $x$ and $r_{Y}^{X}: \mathbf{P i c}^{\text {loc }}(x, X) \rightarrow \mathbf{P i c}^{\text {loc }}(y, Y)$ the natural pull-back map.

Assume that $\pi(Y)$ contains the support of a complete intersection subscheme $x \subset Z \subset X$ of dimension at least 3 . Then

$$
\left(r_{Y}^{X}\right)^{-1} \mathbf{P i c}^{\mathrm{loc}-\tau}(x, X)=\mathbf{P i c}^{\mathrm{loc}-\tau}(y, Y) .
$$

1.5 (Local Néron-Severi groups). One can restate the above result in terms of the local NéronSeveri groups

$$
\operatorname{NS}^{\text {loc }}(x, X):=\mathbf{P i c}^{\mathrm{loc}}(x, X) / \mathbf{P i c} \mathbf{c}^{\mathrm{loc}-\circ}(x, X) .
$$

The local Néron-Severi group is a finitely generated abelian group if char $k=0$, but only the prime-to- $p$ part is known to be finitely generated if char $k=p>0$; see Definition 3.4. Theorem 1.4 is equivalent to saying that the kernel of the natural pull-back map $\mathrm{NS}^{\text {loc }}(x, X) \longrightarrow \mathrm{NS}^{\text {loc }}(y, Y)$ is torsion.

The global variant of this was proved in [Kle66, Remark 3, p. 305].

Theorem 1.4 implies that $\operatorname{ker} r_{Y}^{X} \subset \mathbf{P i c}^{\text {loc- }-\tau}(x, X)$, but for both questions considered above we can say more. Our answers are optimal in characteristic 0 but in characteristic $p>0$ I have not been able to exclude a possibly infinite, discrete, $p^{\infty}$-torsion quotient. The precise statements are the following.

Theorem 1.6. Assume that $(x, X)$ satisfies Condition 1.3, in particular $\operatorname{dim} X \geqslant 3$. Let $\pi: Y \rightarrow X$ be a finite surjection, $x \subset X$ a 0 -dimensional closed subscheme and $y \subset Y$ the preimage of $x$. Then the kernel of the pull-back map $\pi^{*}: \mathbf{P i c}^{\mathrm{loc}}(x, X) \longrightarrow \mathbf{P i c}^{\mathrm{loc}}(y, Y)$ is

(i) a linear algebraic group if char $k=0$ and

(ii) the extension of a discrete $p^{\infty}$-torsion group by a linear algebraic group if char $k=p>0$.

I do not know any example where the kernel of $\pi^{*}$ is not a linear algebraic group.

We have to be careful with the scheme-theoretic formulation of Question 1.2 since $D \subset X$ need not be $S_{2}$; thus Pic $^{\text {loc }}(x, D)$ need not exist. Nonetheless, for now we use the suggestive notation $r_{D}^{X}: \mathbf{P i c}^{\mathrm{loc}}(x, X) \longrightarrow \mathbf{P i c}^{\mathrm{loc}}(x, D)$; see Definition 3.3 for its precise meaning. 


\section{MAPS BeTWEen LOCAL PiCARD GROUPS}

Theorem 1.7. Assume that $(x, X)$ satisfies Condition 1.3 and $\operatorname{dim} X \geqslant 4$. Let $x \subset D \subset X$ be an effective Cartier divisor. Then the kernel of the restriction $\operatorname{map} r_{D}^{X}: \mathbf{P i c}^{\text {loc }}(x, X) \longrightarrow \mathbf{P i c}^{\text {loc }}(x, D)$ is

(i) a unipotent algebraic group if char $k=0$,

(ii) a unipotent algebraic group if $X$ is normal and

(iii) an extension of a discrete $p^{\infty}$-torsion group by a unipotent algebraic group if char $k=p>0$.

As before, I do not know any example where the kernel of $r_{D}^{X}$ is not unipotent.

Remark 1.8. In both cases the dimension restrictions are optimal. For example, for the nonnormal surface $S:=(x y z=0) \subset \mathbb{A}^{3}$ with normalization $\pi: \bar{S} \rightarrow S$ we have

$$
\operatorname{ker}\left[\operatorname{Pic}^{\text {loc }}(0, S) \stackrel{\pi^{*}}{\longrightarrow} \mathbf{P i c}^{\text {loc }}(\overline{0}, \bar{S})\right] \cong \mathbb{Z}^{3} .
$$

For the ordinary 3-fold node $X:=\left(x^{2}+y^{2}+z^{2}+t^{2}=0\right) \subset \mathbb{A}^{4}$ and $D:=(t=0)$ we have

$$
\operatorname{ker}\left[\mathbf{P i c}^{\mathrm{loc}}(x, X) \stackrel{r_{D}^{X}}{\longrightarrow} \mathbf{P i c}^{\mathrm{loc}}(x, D)\right] \cong \mathbb{Z} .
$$

There are also many instances where the kernels in Theorem 1.6(1) and Theorem 1.7(1) are positive dimensional; see Examples 4.3 and 4.7, [Kol13a, Example 12] and [BdJ14, Examples 1.31$35]$.

It is also important to assume in Theorem 1.7 that $D$ be a Cartier divisor. For instance, let $H \subset \mathbb{P}^{n}$ be a hyperplane and $P \subset \mathbb{P}^{n} \backslash H$ a finite set of points. Let $(v, X)$ be a cone over the blow-up $B_{P} \mathbb{P}^{n}$ with vertex $v$ and $v \in D \subset X$ the cone over $H$. Then

$$
\operatorname{ker}\left[\mathbf{P i c}^{\mathrm{loc}}(v, X) \stackrel{r_{D}^{X}}{\longrightarrow} \mathbf{P i c}^{\mathrm{loc}}(v, D)\right] \cong \mathbb{Z}^{|P|} .
$$

If $\operatorname{depth}_{x} X \geqslant 3$, then $\mathbf{P i c}{ }^{\text {loc-o }}(x, X)$ is trivial and we get the following results.

THEOREM 1.9. We take the notation and assumptions of Theorems 1.6-1.7. Assume in addition that $\operatorname{depth}_{x} X \geqslant 3$. Then

(i) $r_{D}^{X}: \mathbf{P i c}^{\mathrm{loc}}(x, X) \longrightarrow \mathbf{P i c}^{\mathrm{loc}}(x, D)$ is injective,

(ii) $\operatorname{ker}\left(\pi^{*}\right)$ is finite if char $k=0$ and

(iii) the prime-to-p part of $\operatorname{ker}\left(\pi^{*}\right)$ is finite if char $k=p>0$.

1.10 (Numerical criteria for Cartier divisors). Let $T$ be an irreducible, regular, 1-dimensional scheme and $f: X \rightarrow T$ a flat, projective morphism of relative dimension $n$. Assume for simplicity that $f$ has normal fibers.

Let $D$ be a divisor on $X$ such that $D_{t}:=\left.D\right|_{X_{t}}$ is Cartier for every $t \in T$. In general $D$ need not be Cartier. For example, let

$$
X=\left(x^{2}-y^{2}+z^{2}-t^{2}=0\right) \subset \mathbb{A}_{x y z}^{3} \times \mathbb{A}_{t} \quad \text { and } \quad D=(x-y=z-t=0) .
$$

The divisor $D$ is Cartier, except at the origin, where it is not even $\mathbb{Q}$-Cartier. However, $D_{0}$ is a line on a quadric cone, hence $2 D_{0}=(x-y=0)$ is Cartier. Thus $2 D$ is Cartier on every fiber, but it is not Cartier.

We discuss several criteria in Section 11. The following is an easy-to-state special case of Theorem 11.6. 


\section{J. KOLlÁR}

THEOREM 1.11. Using the above notation, assume in addition that $D_{t}$ is ample for every $t \in T$. Then $D$ is a Cartier divisor on $X$ if and only if the self-intersection number $\left(D_{t}^{n}\right)$ is independent of $t \in T$.

1.12 (Complex analytic case). Even if $k=\mathbb{C}$, the proofs of Theorems 1.6-1.7 proceed by reduction to positive characteristic. There are nonisolated complex analytic singularities that do not lie on any algebraic variety. Our proof does not apply to them, but the conclusions are hopefully valid.

Over $\mathbb{C}$ one can use the first Chern class to realize $\mathrm{NS}^{\text {loc }}(x, X)$ as a subgroup of $H^{2}(\operatorname{link}(x, X)$, $\mathbb{Z})$, the second cohomology of the link of $(x, X)$; see $\S 5.2$. Thus it would be natural to try to prove the analytic case by showing that the kernels of the corresponding maps between these cohomology groups are torsion. This is, however, not true.

Example 5.5 shows a semi-log canonical hypersurface singularity $(x, X)$ of dimension 3 whose normalization $\pi:(\bar{x}, \bar{X}) \rightarrow(x, X)$ is also a hypersurface singularity and for which

$$
\operatorname{ker}\left[\pi^{*}: H^{2}(\operatorname{link}(x, X), \mathbb{Z}) \longrightarrow H^{2}(\operatorname{link}(\bar{x}, \bar{X}), \mathbb{Z})\right] \cong \mathbb{Z}^{2} .
$$

For Theorem 1.7, similar examples are given in [Kol13a, Section 5].

\section{Outline of the proofs}

The proofs of Theorems 1.4-1.9 have four major components:

- the proof of Theorem 1.7 when $X$ is normal and char $k>0$ (This was done in [BdJ14].)

- the proof of Theorem 1.6 over finite fields (This is done in Section 6.)

(These two together imply Theorem 1.4 over finite fields.)

- a lifting argument that derives the general assertions from the finite field cases (This is done in Sections 7-9.)

- the treatment of torsion elements in $\operatorname{ker} r_{D}^{X}$. (The nonexistence of prime-to- $p$ torsion follows from [Gro68, XIII.2.1]; see Paragraph 2.5. The $p^{\infty}$-torsion is excluded in $\S 10.4$.)

The key to the results is to find a good answer to the following.

Question 2.1. Let $(x, X)$ be a local scheme and $L$ a line bundle on $X \backslash\{x\}$. How can one check if $L$ is in $\mathbf{P i c}^{\operatorname{loc}-\tau}(x, X)$ ?

In the global case, when $Y$ is a proper scheme over a field $k$, there is a simple numerical criterion: a line bundle $L$ is in $\mathbf{P i c}^{\tau}(Y)$ if and only if $L$ has degree 0 on every reduced, irreducible curve $C \subset Y$.

For the local Picard group there are no proper curves to work with and I do not know any similar numerical criterion to identify $\mathbf{P i c}^{\text {loc-o}}(x, X)$ or $\mathbf{P i c}{ }^{\text {loc- }-\tau}(x, X)$ in general. (If $X$ is normal and it has a resolution of singularities $X^{\prime} \rightarrow X$, then one can work on $X^{\prime}$ and use the exceptional curves. This was used in [Bou78] to prove that $\mathrm{NS}^{\text {loc }}(x, X)$ is finitely generated.)

Over a finite field the $\mathbb{F}_{q}$-points of a finite-type group scheme form a finite group, which gives the following.

Claim 2.2. Let $(x, X)$ be a local scheme over a finite field $\mathbb{F}_{q}$ that satisfies Condition 1.3, and let $L$ be a line bundle on $X \backslash\{x\}$. Then $L \in \mathbf{P i c}^{\text {loc- }-\tau}(x, X)$ if and only if $L$ is torsion.

This leads to a somewhat roundabout way of proving that a line bundle $L$ is in the connected component of a Picard group: 


\section{MAPS BetWeEN LOCAL PiCARD GRoups}

- Reduce everything to finite fields.

- Check that we get torsion line bundles.

- Lift back to the original setting.

The last step is the critical one; let us consider it in more detail for local schemes $(x, X)$ of finite type over a field $k$. We may assume that $k$ is finitely generated over its prime field, thus we can view $k$ as the function field of an integral scheme $S$ that is of finite type over $\mathbb{Z}$. By a suitable choice of $S$ we may even assume that we have

(i) a scheme flat and of finite type $X_{S} \rightarrow S$ with a section $\sigma: S \rightarrow X_{S}$ and

(ii) a line bundle $L_{S}$ on $X_{S} \backslash \sigma(S)$ such that over the generic fiber we recover $(x, X)$ and $L$.

The key technical result that we need is the following.

Claim 2.3. We have $L \in \mathbf{P i c}^{\operatorname{loc}-\tau}(x, X)$ if and only if the set of closed points

$$
\left\{s \in S \text { such that }\left.L_{S}\right|_{X_{s}} \text { is torsion }\right\}
$$

is Zariski dense in $S$.

This is quite easy to prove if the local Picard groups of the fibers $\left(x_{s}, X_{s}\right)$ are themselves fibers of a "reasonable" group scheme $\mathbf{P i c}_{S}^{\text {loc }}(\sigma, X)$. Even in the proper case, such relative Picard groups exist only under some restrictions; see [BLR90, Chapter 8] for a detailed discussion. In the local case, the existence of such a group scheme $\mathbf{P i c}_{S}^{\text {loc }}(\sigma, X)$ is not known. We prove that, at least after replacing $S$ by a dense open subset of $\operatorname{red} S$, there is a good-enough approximation of $\mathbf{P i c}{ }_{S}^{\text {loc-o }}(\sigma, X)$ to make the rest of the proof work; see Section 9.

Constructing $\mathbf{P i c}_{S}^{\text {loc-o }}(\sigma, X)$ is relatively easy if the generic point $s_{g} \in S$ has characteristic 0 , since then Pic ${ }^{\text {loc-o}}\left(x_{g}, X_{g}\right)$ is a smooth algebraic group. However, in positive characteristic we need to understand the obstruction theory of the Picard functor. A delicate technical point is that the obstruction theory is governed by $H_{x}^{2}\left(X, \mathcal{O}_{X}\right)$, which is usually infinite dimensional. A section of a coherent sheaf over $S$ vanishes at the generic point if and only if it vanishes at a Zariski-dense set of closed points, but this is no longer true for quasi-coherent sheaves; see Example 10.1. Thus the general theory does not exclude the possibility that the dimension of $\mathbf{P i c}{ }^{\text {loc-o }}\left(x_{s}, X_{s}\right)$ jumps at every closed point.

Once this issue is settled, we complete the proofs as follows.

Since $S$ is of finite type over $\mathbb{Z}$, the residue fields at closed points are all finite.

Over $\mathbb{F}_{q}$ we prove Theorem 1.6 for the normalization $\pi: \bar{X} \rightarrow X$ by factoring it as

$$
\pi: \bar{X} \stackrel{\pi_{3}}{\longrightarrow} X^{\mathrm{wn}} \stackrel{\pi_{2}}{\longrightarrow} \operatorname{red} X \stackrel{\pi_{1}}{\longrightarrow} X,
$$

where $X^{\mathrm{wn}}$ is the weak normalization of $X$. The nontrivial part is the finiteness of the kernel for $\pi_{3}^{*}$. This is established in Proposition 6.12, using the quotient theory of [Kol12] and Seifert $\mathbb{G}_{m}$-bundles as in [Kol13b, 9.53]. Besides proving Theorem 1.6 over $\mathbb{F}_{q}$, this reduces the proof of Theorem 1.7 in positive characteristic to the case when $X$ is normal. The latter was done in [BdJ14].

For all the theorems, we use Claim 2.3 to pass to arbitrary base fields $k$.

2.4. Here we show that Theorem 1.6 is implied by the special case when $Y=\bar{X}$. To see this, let $\sigma: \bar{Y} \rightarrow Y$ be the normalization of $Y$. It is enough to show that the kernel of the composition

$$
\operatorname{Pic}^{\mathrm{loc}}(x, X) \stackrel{\pi^{*}}{\longrightarrow} \mathbf{P i c}^{\mathrm{loc}}(y, Y) \stackrel{\sigma^{*}}{\longrightarrow} \mathbf{P i c}^{\mathrm{loc}}(\bar{y}, \bar{Y})
$$




\section{J. Kollár}

is contained in $\mathbf{P i c}^{\text {loc- }-\tau}(x, X)$. The map $\sigma^{*} \circ \pi^{*}$ is also the composition of

$$
\operatorname{Pic}^{\mathrm{loc}}(x, X) \stackrel{\pi^{*}}{\longrightarrow} \operatorname{Pic}^{\mathrm{loc}}(\bar{x}, \bar{X}) \stackrel{\bar{\pi}^{*}}{\longrightarrow} \mathbf{P i c}^{\mathrm{loc}}(\bar{y}, \bar{Y}) .
$$

We already know that the kernel of $\pi^{*}$ is contained in $\mathbf{P i c}{ }^{\text {loc }-\tau}(x, X)$.

If $q: U \rightarrow V$ is a finite surjection between irreducible normal schemes, then for any line bundle $L$ on $V$ we have $L^{\operatorname{deg} U / V} \cong \operatorname{norm}_{U / V} q^{*} L$. Thus the kernel of $\bar{\pi}^{*}$ is torsion and so the kernel of $\sigma^{*} \circ \pi^{*}=\bar{\pi}^{*} \circ \pi^{*}$ is also contained in $\mathbf{P i c}^{\text {loc- }-\tau}(x, X)$.

2.5. We take the notation and assumptions of Theorem 1.7. Here we show that once we know that $\operatorname{ker}\left(r_{D}^{X}\right) \subset \mathbf{P i c}^{\text {loc- }-\tau}(x, X)$, the remaining claims about unipotence follow.

Let $L \in \operatorname{Pic}^{\text {loc }}(x, X)$ be a nontrivial line bundle such that $\left.L\right|_{D} \cong \mathcal{O}_{D}$ and $L^{m} \cong \mathcal{O}_{X}$ for some $m>0$ not divisible by char $k$. Choose $m$ to be the smallest with these properties. Then $L$ determines a degree $m$, irreducible, cyclic cover $\tau: \tilde{X} \rightarrow X$ that induces a trivial cover on $D \backslash\{x\}$. Thus $\tilde{D}:=\tau^{-1}(D)$ is a Cartier divisor such that $\tilde{D} \backslash\{\tilde{x}\}$ has $m>1$ connected components. By [Gro68, XIII.2.1] this is impossible.

Thus $\operatorname{ker}\left(r_{D}^{X}\right)$ is torsion free if char $k=0$ and contains only $p^{\infty}$-torsion if char $k=p>0$. Using Lemma 2.6, this implies that once $\operatorname{ker}\left(r_{D}^{X}\right)$ is known to be of finite type, it is unipotent if char $k=0$ and unipotent up to $p^{\infty}$-torsion if char $k=p>0$. (Note that a finite $p^{\infty}$-torsion group is unipotent if $\operatorname{char} k=p>0$, so $\operatorname{ker}\left(r_{D}^{X}\right)$ is unipotent unless the $p^{\infty}$-torsion part is not finitely generated.)

Lemma 2.6. Let $G$ be a locally of finite type, commutative algebraic group over a field $k$. Assume that $G$ is torsion free if char $k=0$ and contains only $p^{\infty}$-torsion if char $k=p>0$.

Then $G^{\circ}$ is unipotent and $G / G^{\circ}$ is torsion free if char $k=0$ and contains only $p^{\infty}$-torsion if char $k=p>0$.

Proof. A connected commutative algebraic group $H$ has a unique connected subgroup $0 \subset H_{l} \subset H$ such that $H / H_{l}$ is an Abelian variety and $H_{l} \cong H_{m}+H_{u}$ is a linear algebraic group, where $H_{m}$ is multiplicative and $H_{u}$ is unipotent.

The existence of $H_{l}$ is called Chevalley's theorem; the first published proof is in [Bar55], see [BSU13] for a modern treatment. The decomposition $H_{l} \cong H_{m}+H_{u}$ is in most books on linear algebraic groups; see for instance [Bor91, Theorem 4.7].

Note that both Abelian varieties and multiplicative groups contain many torsion elements. These lift back to torsion elements in $H$ using the following elementary observation.

Let $H$ be a group and $K \subset H$ a central subgroup. Assume that $\bar{h} \in H / K$ is $m$-torsion and that $K$ is $m$-divisible. Then $\bar{h}$ lifts to an $m$-torsion element $h \in H$.

\section{Definition of local Picard groups}

The literature is very inconsistent, there are at least four variants of what authors call the local Picard group.

Definition 3.1 (Local Picard group). Let $X$ be a scheme and $x \in X$ a point. Assume for simplicity that $X$ is excellent and $\operatorname{depth}_{x} \mathcal{O}_{X} \geqslant 2$.

The local Picard group $\operatorname{Pic}^{\text {loc }}(x, X)$ is a group whose elements are $S_{2}$ sheaves $F$ on some neighborhood $x \in U \subset X$ such that $F$ is locally free on $U \backslash\{x\}$. Two such sheaves give the same element if they are isomorphic over some neighborhood of $x$. The product is given by the $S_{2}$-hull of the tensor product. 


\section{MAPS BetWeEN LOCAL PiCARD GRoups}

One can also realize the local Picard group as $\operatorname{Pic}\left(\operatorname{Spec} \mathcal{O}_{x, X} \backslash\{x\}\right)$ or as the direct limit of $\operatorname{Pic}(U \backslash\{x\})$ as $U$ runs through all open Zariski neighborhoods of $x$. Usually it is necessary to take the limit, for example if $X$ is a smooth curve of genus at least 1 .

If $X$ is normal and $X \backslash\{x\}$ is smooth (or locally factorial), then $\operatorname{Pic}^{\text {loc }}(x, X)$ is isomorphic to the divisor class group of $\mathcal{O}_{x, X}$.

In many contexts it is more natural to work with the étale-local Picard group $\operatorname{Pic}^{\text {et-loc }}(x, X):=$ $\operatorname{Pic}\left(\operatorname{Spec} \mathcal{O}_{x, X}^{h} \backslash\{x\}\right)$, where $\mathcal{O}_{x, X}^{h}$ is the Henselization of the local ring $\mathcal{O}_{x, X}$. Alternatively, $\mathrm{Pic}^{\text {et-loc }}(x, X)$ is the direct limit of $\mathrm{Pic}^{\text {loc }}\left(x^{\prime}, X^{\prime}\right)$ as $\left(x^{\prime}, X^{\prime}\right)$ runs through all étale neighborhoods of $(x, X)$. Usually $\mathrm{Pic}^{\text {loc }}\left(x^{\prime}, X^{\prime}\right) \rightarrow \mathrm{Pic}^{\text {et-loc }}(x, X)$ is neither injective nor surjective and it is necessary to take the limit; see Example 4.4 .

Even for isolated singularities over $\mathbb{C}$, it is quite hard to understand the relationship between $\operatorname{Pic}^{\text {loc }}(x, X)$ and $\mathrm{Pic}^{\mathrm{et}-\mathrm{loc}}(x, X)$. By [PS94] there are many singularities such that $\operatorname{Pic}^{\mathrm{loc}}(x, X)=$ 0 , yet $\operatorname{Pic}^{\text {et-loc }}(x, X)$ is large.

Example 4.4 shows that for some rather simple singularities we always have $\operatorname{Pic}^{\text {loc }}(x, X) \neq$ $\operatorname{Pic}^{\text {et-loc }}(x, X)$.

Definition 3.2 (Picard group of a local ring). Let $(R, m)$ be a semilocal ring. Assume for simplicity that $R$ is excellent and $\operatorname{depth}_{m} R \geqslant 2$. We can define its local Picard group $\operatorname{Pic}^{\text {loc }}(R, m)$ purely algebraically as follows. Its elements are isomorphism classes of finite $R$-modules $M$ such that $\operatorname{depth}_{m} M \geqslant 2$ and that $M_{r}$ is locally free of rank 1 over $R_{r}$ for every non-zero-divisor $r \in m$. The product is given by the $S_{2}$-hull of the tensor product.

If $(R, m)$ is the (semi)local ring of a point $x$ (or of a 0 -dimensional subscheme) on a scheme $X$, then $\operatorname{Pic}^{\text {loc }}(R, m)=\operatorname{Pic}^{\text {loc }}(x, X)$.

In particular, $\operatorname{Pic}^{\text {loc }}(x, X)$ does not depend on our choice of the base scheme.

Next, assume that $(x, X)$ is essentially of finite type over $(s, S)$. Then $k(x)$ is a finitely generated field extension of $k(s)$. Pick a transcendence basis $\bar{t}_{1}, \ldots, \bar{t}_{m} \in k(x) / k(s)$, lift it back to $t_{1}, \ldots, t_{m} \in \mathcal{O}_{x, X}$, and localize $\mathcal{O}_{s, S}\left[t_{1}, \ldots, t_{m}\right]$ at the generic point of its intersection with $m_{x}$ to get $\left(m_{R}, R\right)$. We can now view $\mathcal{O}_{x, X}$ as an essentially of finite type $R$-algebra. The advantage is that now $\mathcal{O}_{x, X} / m_{x}$ is a finite extension of $R / m_{R}$. Thus $\mathcal{O}_{x, X}$ is the localization of a finite type $R$-algebra at a closed point.

That is, in the study of local Picard groups on schemes of finite type, it is sufficient to work with closed points.

Definition 3.3 (Local Picard functor and scheme, [Bou78]). Let $k$ be a field and $(x, X)$ a local, Noetherian $k$-scheme. Set $U:=\operatorname{Spec}_{X} \mathcal{O}_{x, X} \backslash\{x\}$.

For a local $k$-algebra $A$, consider the presheaf

$$
A \mapsto \operatorname{Pic}\left(\left(U \times_{k} \operatorname{Spec} A\right)^{h}\right),
$$

where the superscript $h$ denotes the Henselization. Sheafifying in the étale topology gives the local Picard functor Pic ${ }^{\text {loc }}(x, X)$.

Thus the local Picard functor works with objects $(\pi:(\tilde{x}, \tilde{X}) \rightarrow(x, X), \tilde{L})$, where $\pi$ is étale and $\tilde{L}$ is a line bundle on $\tilde{X} \backslash \tilde{x}$.

By [Bou78, Theorem 1.ii], if $\operatorname{depth}_{x} X \geqslant 2$ and $H^{1}\left(U, \mathcal{O}_{U}\right) \cong H_{x}^{2}\left(X, \mathcal{O}_{X}\right)$ is finite dimensional, then the local Picard functor is represented by a $k$-group scheme $\mathbf{P i c}^{\text {loc }}(x, X)$ that is locally of finite type. The tangent space of $\mathbf{P i c}^{\text {loc }}(x, X)$ at the identity is naturally isomorphic to $H_{x}^{2}\left(X, \mathcal{O}_{X}\right)$. 


\section{J. Kollár}

If $H_{x}^{2}\left(X, \mathcal{O}_{X}\right)=0$, then $\mathbf{P i c}^{\text {loc }}(x, X)$ is essentially the same as $\mathrm{NS}^{\text {loc }}(x, X)$. The interesting case is when $0<\operatorname{dim}_{k} H_{x}^{2}\left(X, \mathcal{O}_{X}\right)<\infty$. This holds if

(1.a) $k(x)$ is finite over $k$,

(1.b) $X$ is $S_{2}$ and

(1.c) $X$ is pure of dimension at least 3 .

Note that $H_{x}^{2}\left(X, \mathcal{O}_{X}\right)=0$ if and only if $\operatorname{depth}_{x} \mathcal{O}_{X} \geqslant 3$ and that the conditions (1.a-c) are almost necessary; see [Gro68, VIII.2.3].

Algebraic equivalence is given by

(2.a) a connected $k$-scheme $T$ with two points $t_{1}, t_{2}$,

(2.b) an étale morphism $\pi: Y \rightarrow X \times T$ such that the injection $\{x\} \times T \hookrightarrow X \times T$ lifts to $\sigma:\{x\} \times T \hookrightarrow Y$ and

(2.c) a line bundle $L_{Y}$ on $Y \backslash \sigma(\{x\} \times T)$.

Let a subscript $i$ denote restriction to the fiber over $t_{i}$. The two line bundles

$$
\left(\pi_{1}:\left(y_{1}, Y_{1}\right) \rightarrow(x, X), L_{1}\right) \quad \text { and } \quad\left(\pi_{2}:\left(y_{2}, Y_{2}\right) \rightarrow(x, X), L_{2}\right)
$$

are declared algebraically equivalent. The following elementary property is quite useful.

(3) Two line bundles $L_{1}, L_{2} \in \operatorname{Pic}^{\text {loc }}(x, X)$ are algebraically equivalent if and only if they are algebraically equivalent after some field extension $K \supset k$.

All line bundles algebraically equivalent to the trivial bundle $\mathcal{O}_{X}$ form the identity component of $\mathbf{P i c}{ }^{\text {loc }}(x, X)$, denoted by $\mathbf{P i c}{ }^{\text {loc-o }}(x, X)$. As usual, $\mathbf{P i c}{ }^{\text {loc }-\tau}(x, X) \subset \mathbf{P i c}^{\text {loc }}(x, X)$ denotes the union of those components that become torsion elements in $\mathbf{P i c}^{\text {loc }}(x, X) / \mathbf{P i c}^{\text {loc-o }}(x, X)$.

If $H_{x}^{2}\left(X, \mathcal{O}_{X}\right)$ is infinite dimensional, then $\mathbf{P i c}^{\text {loc }}(x, X)$ does not exist. However, as long as $\operatorname{depth}_{x} X \geqslant 2$, the unit section of $\operatorname{Pic}^{\text {loc }}(x, X)$ is represented by a finitely presented closed immersion, by [Bou78, Theorem 1.i]. We can use this to define the kernel used in Theorem 1.7.

Thus assume that $X$ is $S_{2}$, that $\operatorname{dim} X \geqslant 4$ and that $x \in D \subset X$ is a Cartier divisor. Set $\tilde{D}:=\operatorname{Spec}_{D} j_{*} \mathcal{O}_{D \backslash x}$, and let $\tilde{x} \subset \tilde{D}$ be the preimage of $x$. Then $\operatorname{depth}_{\tilde{x}} \tilde{D} \geqslant 2$; hence, by [Bou78, Theorem 1.i], there is a subgroup scheme $\mathbf{K}(X \mid D) \subset \mathbf{P i c}^{\text {loc }}(x, X)$ representing those line bundles that become trivial when restricted to $D \backslash\{x\} \cong \tilde{D} \backslash\{\tilde{x}\}$. This $\mathbf{K}(X \mid D)$ gives the precise definition of $\operatorname{ker}\left[r_{D}^{X}: \mathbf{P i c}^{\mathrm{loc}}(x, X) \longrightarrow \mathbf{P i c}^{\mathrm{loc}}(x, D)\right]$ used in Theorem 1.7.

Definition 3.4 (Local Néron-Severi group). Let $k$ be a field and $(x, X)$ a local, Noetherian $k$-scheme such that $\mathbf{P i c}^{\text {loc }}(x, X)$ exists. The quotient

$$
\operatorname{NS}^{\text {loc }}(x, X):=\mathbf{P i c}^{\text {loc }}(x, X) / \mathbf{P i c}^{\text {loc-o}}(x, X)
$$

is called the local Néron-Severi group. The analytic methods show that if $k$ has characteristic 0 , then $\mathrm{NS}^{\text {loc }}(x, X)$ is a finitely generated abelian group; see Proposition 5.3. Combining the method of [Bou78] with [dJ96], we see that, even in characteristic $p>0$, the local Néron-Severi group is finitely generated if $X$ is normal and finitely generated modulo $p^{\infty}$-torsion in general. Our computations show that the $p^{\infty}$-torsion part has bounded exponent, but say nothing about finite generation. I do not know any examples satisfying Condition 1.3 where $\operatorname{NS}^{\text {loc }}(x, X)$ is not finitely generated.

The above is probably not a completely agreed-upon definition; another candidate is the usually smaller

$$
\operatorname{Pic}^{\mathrm{loc}}(x, X) /\left(\operatorname{Pic}^{\mathrm{loc}}(x, X) \cap \mathbf{P i c}^{\mathrm{loc}-\circ}(x, X)\right) .
$$




\section{MAPS BeTWEen LOCAL PiCARD GROUPS}

3.5 (Comparing Pic and Pic). Let $(x, X)$ be a local, Noetherian scheme over a field $k$. By definition there is a natural injection

$$
\operatorname{Pic}^{\mathrm{loc}}(x, X) \hookrightarrow \mathbf{P i c}^{\mathrm{loc}}(x, X)(k),
$$

which is usually not a surjection. If $k$ is algebraically closed, then, essentially by definition,

$$
\mathrm{Pic}^{\mathrm{et}-\mathrm{loc}}(x, X) \cong \mathbf{P i c}^{\mathrm{loc}}(x, X)(k) .
$$

If, in addition, $X$ is complete or henselian, then

$$
\operatorname{Pic}^{\text {loc }}(x, X) \cong \mathbf{P i c}^{\text {loc }}(x, X)(k) .
$$

\section{Examples of local Picard groups}

Here we discuss a series of examples of local Picard groups. They show that the assumptions of Theorems 1.6 and 1.7 are essentially optimal.

Example 4.1. Let $(0, X)$ be a local scheme with an isolated singularity with a resolution $p: Y \rightarrow X$ that is an isomorphism over $X \backslash\{0\}$. Let $\left\{E_{i}: i \in I\right\}$ be the exceptional divisors. There is a natural exact sequence

$$
0 \rightarrow \sum_{i} \mathbb{Z}\left[E_{i}\right] \rightarrow \operatorname{Pic}(Y) \rightarrow \operatorname{Pic}^{\text {loc }}(0, X) \rightarrow 0
$$

(Left exactness follows from [KM98, 3.39], but there are many other ways to prove it.)

Assume in addition that there is an effective, exceptional divisor $E$ such that $\mathcal{O}_{Y}(-E)$ is $p$-ample. (Any resolution obtained by repeatedly blowing up subschemes whose support lies over $\{0\}$ has this property.) Let $L$ denote the line bundle $\left.\mathcal{O}_{X}(-E)\right|_{E}$; it is ample by assumption.

Let $n E$ denote the subscheme of $X$ defined by $\mathcal{O}_{X}(-n E)$. There is an exact sequence

$$
0 \rightarrow L^{n} \stackrel{h \mapsto 1+h}{\longrightarrow} \mathcal{O}_{(n+1) E}^{*} \rightarrow \mathcal{O}_{n E}^{*} \rightarrow 1
$$

Since $L$ is ample, $H^{1}\left(E, L^{n}\right)=H^{2}\left(E, L^{n}\right)$ for $n \gg 1$. Thus the natural restriction maps $\operatorname{Pic}((n+1) E) \rightarrow \operatorname{Pic}(n E)$ are isomorphisms for $n \gg 1$. If $\mathcal{O}_{X}$ is complete, then

$$
\operatorname{Pic}(Y) \cong \operatorname{Pic}(n E) \text { for } n \gg 1 \text {. }
$$

(This probably needs Grothendieck's existence theorem [Gro60, III.5.1.4].) Thus

$$
\operatorname{Pic}(0, X)=\operatorname{Pic}(n E) / \sum_{i} \mathbb{Z}\left[E_{i}\right] \quad \text { for } \quad n \gg 1 \text {. }
$$

For normal surfaces over a field of characteristic 0, the equalities (4.1.4) are used in [Mum61] to define a local Picard scheme. For $\operatorname{dim} X \geqslant 3$ it is an alternate way of constructing the local Picard scheme. Note, however, that the scheme structures of the two sides can differ in positive characteristic, as we see next.

Example 4.2 (Cones). Let $W \subset \mathbb{P}_{k}^{N}$ be a smooth projective variety and $(0, X)$ the affine cone over $W$; see [Kol13b, 3.8] for our conventions on cones. By blowing up the vertex we get a resolution $p: Y \rightarrow X$, where

$$
X=\operatorname{Spec}_{k} \sum_{r \geqslant 0} H^{0}\left(W, \mathcal{O}_{W}(r)\right) \quad \text { and } \quad Y=\operatorname{Spec}_{W} \sum_{r \geqslant 0} \mathcal{O}_{W}(r) .
$$




\section{J. KOLLÁR}

Thus (4.1.3) suggests that

$$
\operatorname{dim} T_{0} \operatorname{Pic}(Y) \stackrel{?}{=} H^{1}\left(Y, \mathcal{O}_{Y}\right)=\sum_{r \geqslant 0}^{\infty} H^{1}\left(W, \mathcal{O}_{W}(r)\right) .
$$

On the other hand, $\operatorname{dim} T_{0} \operatorname{Pic}^{\text {loc }}(0, X)$ equals $H^{1}\left(U, \mathcal{O}_{U}\right)$, where $U=X \backslash\{0\}$. Since $U=$ $\operatorname{Spec}_{W} \sum_{-\infty}^{\infty} \mathcal{O}_{W}(r)$, we see that

$$
\operatorname{dim} T_{0} \mathbf{P i c}^{\mathrm{loc}}(0, X)=\sum_{-\infty}^{\infty} H^{1}\left(W, \mathcal{O}_{W}(r)\right) .
$$

Comparing this with (4.2.1), we see extra summands for $r<0$. This is not a problem in characteristic 0 , where these vanish by Kodaira's theorem. However, the two formulas can give different answers in positive characteristic.

Next, assume that the characteristic is 0 . Then the above considerations give an exact sequence

$$
0 \rightarrow \sum_{r>0} H^{1}\left(W, \mathcal{O}_{W}(r)\right) \rightarrow \mathbf{P i c}^{\mathrm{loc}}(0, X) \rightarrow \mathbf{P i c}(W) / \mathbb{Z}\left[\mathcal{O}_{W}(1)\right] \rightarrow 0
$$

Let $H \subset W$ a smooth hyperplane section and $D \subset X$ the cone over it. A similar computation gives an exact sequence

$$
0 \rightarrow \sum_{r>0} H^{1}\left(H, \mathcal{O}_{H}(r)\right) \rightarrow \mathbf{P i c}^{\mathrm{loc}}(0, D) \rightarrow \mathbf{P i c}(H) / \mathbb{Z}\left[\mathcal{O}_{H}(1)\right] \rightarrow 0
$$

Thus, we see that the restriction map $\mathbf{P i c}^{\text {loc }}(0, X) \rightarrow \mathbf{P i c}^{\text {loc }}(0, D)$ has a positive-dimensional kernel if the maps $H^{1}\left(W, \mathcal{O}_{W}(r)\right) \rightarrow H^{1}\left(H, \mathcal{O}_{H}(r)\right)$ are not all injective; cf. [BdJ14, Examples $1.31-35]$.

Example 4.3 (Weighted cones). In Example 4.2, let $(t=0)$ be an equation of $H \subset W$. We can view $t$ as a map $X \rightarrow \mathbb{A}^{1}$. After base change to $t=s^{m}$ we get a new singularity $\left(0, X_{m}\right)$ containing the same $(0, D)$ as a hyperplane section. Thinking of $X$ as $\operatorname{Spec}_{k} \sum_{r \geqslant 0} t^{r} H^{0}\left(W, \mathcal{O}_{W}(r)\right)$, we now have

$$
X_{m}=\operatorname{Spec}_{k} \sum_{r \geqslant 0} s^{r} H^{0}\left(W, \mathcal{O}_{W}(\lfloor r / m\rfloor)\right) .
$$

Computing as in Example 4.2, we get

$$
H_{0}^{2}\left(X_{m}, \mathcal{O}_{X_{m}}\right) \cong \sum_{-\infty}^{\infty} H^{1}\left(W, \mathcal{O}_{W}(\lfloor r / m\rfloor)\right) \cong \bigoplus_{1}^{m} H_{0}^{2}\left(X, \mathcal{O}_{X}\right)
$$

Thus, if char $k=0$, then $\operatorname{dim} \mathbf{P i c}{ }^{\text {loc }}\left(0, X_{m}\right)=m \cdot \operatorname{dim} \mathbf{P} \mathbf{i c}^{\mathrm{loc}}(0, X)$ and so

$$
\operatorname{dim} \operatorname{ker}\left[\mathbf{P i c}^{\mathrm{loc}}\left(0, X_{m}\right) \rightarrow \mathbf{P i c}^{\mathrm{loc}}(0, D)\right]
$$

grows with $m$, save when $\mathbf{P i c}^{\text {loc }}(0, X)$ is 0 dimensional.

Example 4.4. We give examples of normal surface singularities $\left(0, S^{h}\right)$ such that $\mathrm{Pic}{ }^{\text {et-loc }}\left(0, S^{h}\right)$ is positive dimensional but $\operatorname{Pic}^{\text {loc }}(0, S)$ is finitely generated for every algebraic model $S$ of $S^{h}$.

Let $S$ be a normal, projective surface with a single isolated singularity at $(0, S)$. Assume that $\operatorname{Pic}^{\text {loc-o}}(0, S)$ has no Abelian subvarieties. We claim that the image $\operatorname{Pic}(S \backslash 0) \rightarrow \operatorname{Pic}^{\text {loc }}(0, S)$ is finitely generated. 


\section{MAPS BeTWEen LOCAL PiCARD GROUPS}

To see this, let $\pi: T \rightarrow S$ be a resolution of singularities. Then $\operatorname{Pic}(T) \rightarrow \operatorname{Pic}(S \backslash 0)$ is onto

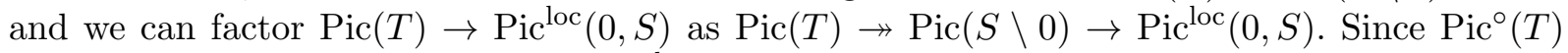
is an Abelian variety, its image in $\operatorname{Pic}^{\text {loc }}(0, S)$ is also an Abelian variety, thus trivial. Hence $\operatorname{Pic}^{\circ}(T) \rightarrow \operatorname{Pic}^{\text {loc-o}}(0, S)$ is the constant map and so $\operatorname{Pic}(T) \rightarrow \operatorname{Pic}^{\text {loc }}(0, S)$ factors through $\mathrm{NS}(T) \rightarrow \operatorname{Pic}^{\text {loc }}(0, S)$.

Here are some concrete equations with the above properties.

(4.4.1) Cusps, for example $\left(x y z+x^{4}+y^{4}+z^{4}=0\right)$ or $\left(z^{2}=x^{2}\left(x^{2}+y^{2}\right)+y^{5}\right)$, have $\mathbf{P i c}^{\mathrm{loc}-\circ}(0, S) \cong \mathbb{G}_{m}$.

(4.4.2) Let $C$ be an irreducible curve with a single node whose normalization $E:=\bar{C}$ is a smooth elliptic curve. There is an extension

$$
1 \rightarrow \mathbb{G}_{m} \rightarrow \operatorname{Pic}^{\circ}(C) \rightarrow \operatorname{Pic}^{\circ}(E) \cong E \rightarrow 0 .
$$

The extension is nonsplit; even more, $\operatorname{Pic}^{\circ}(C)$ does not contain any compact curves if and only if the difference of the two preimages of the node is nontorsion on $\operatorname{Pic}(E)$. For example, the exceptional divisor of the minimal resolution of $\left(z^{2}=x^{2}\left(x^{4}+c y^{4}\right)+y^{7}\right)$ is such a curve; the nontorsion condition holds for very general $c \in \mathbb{C}$.

In the nonnormal case, several new phenomena occur.

Example 4.5 (Nonnormal surfaces). We compute the local Picard group for three of the simplest nonnormal surfaces. Let $S:=(x y=0) \subset \mathbb{A}^{3}$ be the union of two planes in $\mathbb{A}^{3}$, let $T:=\left(z^{2}=\right.$ $0) \subset \mathbb{A}^{3}$ be the double plane in $\mathbb{A}^{3}$, and let $W:=\left(y^{2}-x^{3}=0\right) \subset \mathbb{A}^{3}$ be the product of a cuspidal cubic with a line. The schemes $S, T$ and $W$ are all $S_{2}$.

Let $s \in S$ denote the origin; then $\operatorname{depth}_{s} \mathcal{O}_{S}=2$. The scheme $S$ has two irreducible components, $S_{x}=(x=0) \cong \mathbb{A}^{2}$ and $S_{y}=(y=0) \cong \mathbb{A}^{2}$. The normalization $\bar{S}$ is the disjoint union $S_{x} \amalg S_{y}$. Thus $\operatorname{Pic}^{\text {loc }}(\bar{s}, \bar{S}) \cong 0$. We claim that

$$
\operatorname{Pic}^{\text {loc }}(s, S) \cong \operatorname{ker}\left[\operatorname{Pic}^{\text {loc }}(s, S) \stackrel{\pi^{*}}{\rightarrow} \operatorname{Pic}^{\text {loc }}(\bar{s}, \bar{S})\right] \cong \mathbb{Z} .
$$

To see this, let $L$ be an invertible sheaf on $S \backslash s$. Both $\left.L\right|_{S_{x}}$ and $\left.L\right|_{S_{y}}$ are trivial; let $\sigma_{x}$ and $\sigma_{y}$ be generating sections. Then $\sigma_{x}$ and $\sigma_{y}$ restrict to global sections of $L$ on the punctured $z$-axis. Their quotient is a regular function on the punctured $z$-axis; its order of pole or zero at the origin gives the isomorphism $\operatorname{Pic}^{\text {loc }}(s, S) \cong \mathbb{Z}$.

Next, let $t \in T$ denote the origin; then $\operatorname{depth}_{t} \mathcal{O}_{T}=2$. The normalization of $T$ is $\bar{T}=(z=$ 0) $\subset \mathbb{A}^{3}$. There is an exact sequence

$$
0 \rightarrow \mathcal{O}_{\bar{T} \backslash t} \stackrel{e}{\rightarrow} \mathcal{O}_{T \backslash t}^{*} \rightarrow \mathcal{O}_{\bar{T} \backslash t}^{*} \rightarrow 1
$$

where $e(g)=1+z g$. Taking cohomology and using that $\operatorname{Pic}^{\text {loc }}(t, \bar{T})=0$ gives the isomorphism

$$
\operatorname{Pic}^{\text {loc }}(t, T) \cong H^{1}\left(\bar{T} \backslash t, \mathcal{O}_{\bar{T} \backslash t}\right) \text {. }
$$

The latter can be naturally identified with $\sum_{m \geqslant 1} H^{1}\left(\mathbb{P}^{1}, \mathcal{O}_{\mathbb{P}^{1}}(-m)\right)$. Thus

$$
\begin{aligned}
\operatorname{Pic}^{\mathrm{loc}}(t, T) & \cong \operatorname{ker}\left[\operatorname{Pic}^{\mathrm{loc}}(t, T) \stackrel{\pi^{*}}{\rightarrow} \operatorname{Pic}^{\mathrm{loc}}(\bar{t}, \bar{T})\right] \\
& \cong \text { (infinite dimensional vector space) } .
\end{aligned}
$$

Let $w \in W$ denote the origin. We can write $W$ as $\operatorname{Spec} k\left[t^{2}, t^{3}, z\right]$ with normalization $\bar{W} \cong$ Spec $k[t, z]$. Let $C \subset W$ denote the $z$-axis; this is the singular locus of $W$. There is an exact sequence

$$
1 \rightarrow \mathcal{O}_{W}^{*} \rightarrow \mathcal{O}_{\bar{W}}^{*} \stackrel{d}{\longrightarrow} \mathcal{O}_{C} \rightarrow 0
$$




\section{J. KOLLÁR}

where $d\left(f_{0}(z)+f_{1}(z) t+\cdots\right)=f_{1}(z) / f_{0}(z)$. Then we have the exact sequence

$$
H^{0}\left(\bar{W} \backslash\{w\}, \mathcal{O}_{\bar{W}}^{*}\right) \stackrel{d}{\longrightarrow} H^{0}\left(C \backslash\{w\}, \mathcal{O}_{C}\right) \rightarrow \operatorname{Pic}(W \backslash\{w\}) \rightarrow \operatorname{Pic}(\bar{W} \backslash\{w\})=1 .
$$

Note that $d$ factors as

$$
H^{0}\left(\bar{W} \backslash\{w\}, \mathcal{O}_{\bar{W}}^{*}\right)=H^{0}\left(\bar{W}, \mathcal{O}_{\bar{W}}^{*}\right) \stackrel{d}{\longrightarrow} H^{0}\left(C, \mathcal{O}_{C}\right) \rightarrow H^{0}\left(C \backslash\{w\}, \mathcal{O}_{C}\right) .
$$

This shows that

$$
\begin{aligned}
\operatorname{Pic}^{\operatorname{loc}}(w \in W) & \cong H_{w}^{1}\left(\mathcal{O}_{C}\right) \cong k\left[z, z^{-1}\right] / k[z] \\
& \cong(\text { infinite dimensional vector space })
\end{aligned}
$$

Example 4.6. As a slight variation of Example 4.5, let $X \subset \mathbb{A}^{2 n+1}$ be the union of the linear spaces $X_{1}=\left(x_{1}=\cdots=x_{n}=0\right)$ and $X_{2}=\left(x_{n+2}=\cdots=x_{2 n+1}=0\right)$. Here $X_{1} \cap X_{2} \cong \mathbb{A}^{1}$ and the normalization is $\bar{X}=X_{1} \amalg X_{2}$. Here $X$ is not $S_{2}$ if $n \geqslant 2$, but $\operatorname{depth}_{x} \mathcal{O}_{X}=2$, where $x \in X$ denotes the origin.

As in the previous example, we see that

$$
\operatorname{Pic}^{\text {loc }}(x, X) \cong \operatorname{ker}\left[\operatorname{Pic}^{\operatorname{loc}}(x, X) \stackrel{\pi^{*}}{\rightarrow} \operatorname{Pic}^{\text {loc }}(\bar{x}, \bar{X})\right] \cong \mathbb{Z} .
$$

Example 4.7 (Deminormal varieties, [Kol13b, Section 5.1]). Fix a ground field $k$, and let $X_{i}$ be cones over the Segre embedding of $\mathbb{P}^{1} \times \mathbb{P}^{n}$ with vertices $v_{i}$. Note that the $X_{i}$ have rational singularities at the origin and $\operatorname{Pic}^{\text {loc }}\left(v_{i}, X_{i}\right) \cong \mathbb{Z}$.

Pick distinct points $p_{j} \in \mathbb{P}^{1}$, and let $D_{i j} \subset X_{i}$ be the cone over $\left\{p_{j}\right\} \times \mathbb{P}^{n}$. Note that $D_{i j} \cong \mathbb{A}^{n+1}$.

The first example, $Y_{2}$, is obtained by gluing $X_{1}$ to $X_{2}$ using the natural identifications $D_{1 j} \cong D_{2 j}$ for $j=1,2$. Then $\bar{Y}_{2}=X_{1} \amalg X_{2}$ and so $\operatorname{Pic}{ }^{\text {loc }}\left(\bar{v}, \bar{Y}_{2}\right) \cong \mathbb{Z}^{2}$. We claim that

$$
\begin{gathered}
\operatorname{Pic}^{\text {loc }}\left(v, Y_{2}\right) \cong k^{*}+\mathbb{Z}^{2} \text { and } \\
\operatorname{ker}\left[\operatorname{Pic}^{\operatorname{loc}}\left(v, Y_{2}\right) \stackrel{\pi^{*}}{\rightarrow} \operatorname{Pic}^{\operatorname{loc}}\left(\bar{v}, \bar{Y}_{2}\right)\right] \cong k^{*} .
\end{gathered}
$$

The extra $k^{*}$ is obtained as follows. Set $D_{i}=D_{i 1}+D_{i 2} \subset X_{i}$. Take the trivial bundles $\mathcal{O}_{X_{i}}$ and an isomorphism

$$
\phi^{0}: \mathcal{O}_{D_{1} \backslash v} \cong \mathcal{O}_{D_{2} \backslash v}
$$

to get an element of $\operatorname{Pic}^{\text {loc }}\left(v, Y_{2}\right)$. Two such isomorphisms give the same line bundle if and only if they differ by multiplication by sections of $H^{0}\left(X_{i} \backslash v, \mathcal{O}_{X_{i} \backslash v}^{*}\right)$. Since $\operatorname{dim} D_{i j} \geqslant 2$, the isomorphism $\phi^{0}$ extends to a pair of isomorphisms

$$
\phi_{j}: \mathcal{O}_{D_{1 j}} \cong \mathcal{O}_{D_{2 j}} \quad \text { for } j=1,2 .
$$

These give isomorphisms

$$
\phi_{j}(v): k \cong k\left(v_{1}\right) \cong k\left(v_{2}\right) \cong k .
$$

The quotient $\phi_{1}(v) / \phi_{2}(v)$ gives a well-defined element of $k^{*}$.

The second example, $Y_{3}$, is obtained by gluing $X_{1}$ to $X_{2}$ using the natural identifications $D_{1 j} \cong D_{2 j}$ for $j=1,2,3$. We claim that here the kernel is a direct product:

$$
\begin{gathered}
\operatorname{Pic}^{\text {loc }}\left(v, Y_{3}\right) \cong k^{n+1} \times\left(k^{*}\right)^{2}+\mathbb{Z}^{2} \text { and } \\
\operatorname{ker}\left[\operatorname{Pic}^{\operatorname{loc}}\left(v, Y_{3}\right) \stackrel{\pi^{*}}{\rightarrow} \operatorname{Pic}^{\operatorname{loc}}\left(\bar{v}, \bar{Y}_{3}\right)\right] \cong k^{n+1} \times\left(k^{*}\right)^{2} .
\end{gathered}
$$




\section{MAPS BetWeEN LOCAL PiCARD GRoups}

To see this, set $D_{i}=D_{i 1}+D_{i 2}+D_{i 3} \subset X_{i}$. The trivial bundles $\mathcal{O}_{X_{i}}$ can be glued using a section of $H^{0}\left(D_{i} \backslash v, \mathcal{O}_{D_{i} \backslash v}^{*}\right)$. Two such sections give the same line bundle if and only if they differ by multiplication by a section of $H^{0}\left(X_{i} \backslash v, \mathcal{O}_{X_{i} \backslash v}^{*}\right)$. The previous considerations explain the $\left(k^{*}\right)^{2}$, but now we also get new conditions from sections of the form $1+$ (linear functions). On $D_{i}$, we have $n+1$ independent linear functions on each $D_{i j}$, giving $3 n+3$ independent linear functions all together. On $X_{i} \subset \mathbb{A}^{2 n+2}$ we only have $2 n+2$ independent linear functions. This accounts for the $k^{n+1}$ part.

In principle, we could get more conditions by considering $1+$ (quadratic functions), but it is easy to see that this does not happen for three or four copies of $D_{i j}$. For more copies, we obtain conditions coming from higher-degree polynomials.

Example 4.8 (Pinch point). Let $k$ be any field and $S:=\left(x^{2}=y^{2} z\right) \subset \mathbb{A}^{3}$ the pinch point. Its normalization is the map $\pi: \bar{S}:=\mathbb{A}_{u v}^{2} \rightarrow S$ given by $(u, v) \mapsto\left(u v, u, v^{2}\right)$. The line $(x=z=0)$ generates $\operatorname{Pic}^{\text {loc }}(0, S) \cong \mathbb{Z} / 2$.

Note that $\pi$ is a homeomorphism if char $k=2$. This suggests that in positive characteristic there may not be a perfect analog of the first Chern class as a mapping to topological cohomology.

A more general version is the following.

Example 4.9. Start with $(\bar{X}, \bar{D}) \cong\left(\mathbb{A}^{n}, \mathbb{A}^{n-1}\right)$, and let $\bar{x} \in \mathbb{A}^{n-1}$ be the origin. Let $\tau: \mathbb{A}^{n-1} \rightarrow$ $\mathbb{A}^{n-1}$ be coordinate-wise multiplication by a primitive $r$ th root of unity. Assume that the characteristic does not divide $r$.

Construct $\pi: \bar{X} \rightarrow X$ by identifying the points in the $\tau$-orbits with one another. Thus $X$ is an affine variety, even Cohen-Macaulay. For $r=2$ it has only double normal crossing singularities along $D:=\pi(\bar{D})$. We claim that

$$
\operatorname{Pic}^{\mathrm{loc}}(x, X)=\operatorname{ker}\left[\operatorname{Pic}^{\mathrm{loc}}(x, X) \stackrel{\pi^{*}}{\rightarrow} \operatorname{Pic}^{\mathrm{loc}}(\bar{x}, \bar{X})\right] \cong \mathbb{Z} / r .
$$

For any $r$ th root of unity $\epsilon$, the $\tau$-action on $\bar{D}$ can be lifted to $\mathcal{O}_{\bar{D}}$ as $g(x) \mapsto \epsilon \cdot g\left(\tau^{-1}(x)\right)$. Taking the quotient, we obtain line bundles $L(\epsilon)$ over $X$ corresponding to the $r$ th roots of unity.

Choose coordinates such that $\bar{D}=\left(z_{n}=0\right)$. A local trivialization of $L(\epsilon)$ would correspond to an invertible function $\phi\left(z_{1}, \ldots, z_{n}\right)$ such that $\phi\left(\epsilon z_{1}, \ldots, \epsilon z_{n-1}, 0\right)=\epsilon \phi\left(z_{1}, \ldots, z_{n-1}, 0\right)$. This would imply $\phi(0, \ldots, 0)=\epsilon \phi(0, \ldots, 0)$; thus $\phi(0, \ldots, 0)=0$ if $\epsilon \neq 1$. Therefore $\phi$ is not invertible near the origin if $n \geqslant 2$.

\section{Analytic local Picard groups}

Definition 5.1 (Analytic local Picard groups). Let $X$ be a complex analytic space and $x \in X$ a point. Assume for simplicity that $\operatorname{depth}_{x} \mathcal{O}_{X} \geqslant 2$.

Let $W \subset X$ be the intersection of $X$ with a small (open) ball around $x$. The analytic local Picard group $\mathrm{Pic}^{\text {an-loc }}(x, X)$ can be defined as in Definition 3.1 using (analytic) $S_{2}$ sheaves on $W$. By [Art69], if $X$ is an algebraic variety over $\mathbb{C}$, then there is a natural isomorphism

$$
\operatorname{Pic}^{\text {et-loc }}(x, X) \cong \operatorname{Pic}^{\text {an-loc }}\left(x, X^{\text {an }}\right) \text {. }
$$

By [Siu69], if $X$ is $S_{2}$ and has pure dimension at least 3, then

$$
\operatorname{Pic}^{\text {an }}(W \backslash\{x\}) \cong \operatorname{Pic}^{\text {an-loc }}(x, X) .
$$

Note that if $\operatorname{dim} X=2$, then $\operatorname{Pic}^{\text {an }}(W \backslash\{x\})$ is infinite dimensional but $\operatorname{Pic}^{\text {an-loc }}(x, W)$ is finite dimensional if $X$ is normal. 


\section{J. KOLLÁR}

5.2 (Exponential sequence). Let $U$ be a complex space. Then $\operatorname{Pic}(U) \cong H^{1}\left(U, \mathcal{O}_{U}^{*}\right)$ and the exponential sequence

$$
0 \rightarrow \mathbb{Z}_{U} \stackrel{2 \pi i}{\longrightarrow} \mathcal{O}_{U} \stackrel{\exp }{\longrightarrow} \mathcal{O}_{U}^{*} \rightarrow 1
$$

gives an exact sequence

$$
H^{1}\left(U, \mathcal{O}_{U}\right) \rightarrow \operatorname{Pic}(U) \stackrel{c_{1}}{\longrightarrow} H^{2}(U, \mathbb{Z}) .
$$

Let $X$ be a complex space and $x \in X$ a point, and set $U:=X \backslash\{x\}$. A piece of the local cohomology exact sequence is

$$
H^{1}\left(X, \mathcal{O}_{X}\right) \rightarrow H^{1}\left(U, \mathcal{O}_{U}\right) \rightarrow H_{x}^{2}\left(X, \mathcal{O}_{X}\right) \rightarrow H^{2}\left(X, \mathcal{O}_{X}\right)
$$

Thus if $X$ is Stein, then we have an isomorphism

$$
H^{1}\left(U, \mathcal{O}_{U}\right) \cong H_{x}^{2}\left(X, \mathcal{O}_{X}\right)
$$

For local questions we should replace $X$ by a contractible open neighborhood $x \in W \subset X$. Then $W \backslash\{x\}$ is homotopy equivalent to $\operatorname{link}(x, X)$, which is the intersection of $X$ with a small sphere centered at $x$. Thus $H^{2}(W \backslash\{x\}, \mathbb{Z})=H^{2}(\operatorname{link}(x, X), \mathbb{Z})$.

Combining the above with [Siu69] and [Art69], we obtain the following well-known result.

Proposition 5.3. Let $(x, X)$ be a $\mathbb{C}$-scheme of finite type that is $S_{2}$ and has pure dimension at least 3. Then taking the first Chern class gives an exact sequence

$$
0 \rightarrow \mathbf{P i c}^{\operatorname{loc}-\circ}(x, X) \rightarrow \mathbf{P i c}^{\operatorname{loc}}(x, X) \stackrel{c_{1}}{\rightarrow} H^{2}(\operatorname{link}(x, X), \mathbb{Z}) .
$$

This suggests a topological way to proving Theorem 1.6 in characteristic 0: we should prove that the kernel of the pull-back map

$$
H^{2}(\operatorname{link}(x, X), \mathbb{Z}) \rightarrow H^{2}(\operatorname{link}(\bar{x}, \bar{X}), \mathbb{Z})
$$

is torsion, where $\bar{X} \rightarrow X$ denotes the normalization. However, Examples 5.4 and 5.5 show that these maps can have nontorsion kernel, even for hypersurface singularities. We start with a projective example and then we take a cone over it to get a local example.

Example 5.4 (Singular K3 surface). Let $g_{4}\left(x_{0}, x_{1}, x_{2}\right)$ be a general quartic form. Then

$$
S:=\left(u^{2}=x_{0}^{2} g_{4}\left(x_{0}, x_{1}, x_{2}\right)\right) \subset \mathbb{P}^{3}(1,1,1,3)
$$

is a K3 surface with a double line $L=\left(x_{0}=u=0\right)$. Its normalization $\pi: \bar{S} \rightarrow S$ is the smooth Del Pezzo surface of degree 2

$$
\bar{S}:=\left(v^{2}=g_{4}\left(x_{0}, x_{1}, x_{2}\right)\right) \subset \mathbb{P}^{3}(1,1,1,2) .
$$

Thus $H^{2}(\bar{S}, \mathbb{Z})=\operatorname{Pic}(\bar{S}) \cong \mathbb{Z}^{8}$.

The preimage of the line $L$ is a smooth elliptic curve $E=\left(x_{0}=0\right)$. We claim that

$$
\operatorname{ker}\left[H^{2}(S, \mathbb{Z}) \stackrel{\pi^{*}}{\rightarrow} H^{2}(\bar{S}, \mathbb{Z})\right] \cong H^{1}(E, \mathbb{Z}) \cong \mathbb{Z}^{2} .
$$

To see this, we start with the short exact sequence defining $Q$,

$$
0 \rightarrow \mathbb{Z}_{L} \rightarrow \pi_{*} \mathbb{Z}_{E} \rightarrow Q \rightarrow 0
$$

which shows that

$$
H^{1}(L, Q) \cong H^{1}(E, \mathbb{Z}) \text { and } H^{2}(L, Q)=0 .
$$

The sheaf $Q$ also sits in the short exact sequence

$$
0 \rightarrow \mathbb{Z}_{S} \rightarrow \pi_{*} \mathbb{Z}_{\bar{S}} \rightarrow Q \rightarrow 0
$$




\section{Maps Between LOCAL PicARd Groups}

and from this we get an exact sequence

$$
0 \rightarrow H^{1}(L, Q) \rightarrow H^{2}(S, \mathbb{Z}) \rightarrow H^{2}(\bar{S}, \mathbb{Z}) \rightarrow H^{2}(L, Q)=0 .
$$

Putting (5.4.2) and (5.4.3) together gives (5.4.1).

Example 5.5. Using the previous notation, set

$$
X:=\left(u^{2}=x_{0}^{2} g_{4}\left(x_{0}, x_{1}, x_{2}\right)\right) \subset \mathbb{A}^{4} .
$$

Its normalization $\pi: \bar{X} \rightarrow X$ is given as

$$
\bar{X}:=\left(v^{2}=g_{4}\left(x_{0}, x_{1}, x_{2}\right)\right) \subset \mathbb{A}^{4} .
$$

Note that $X$ and $\bar{X}$ are weighted homogeneous cones over $S$ and $\bar{S}$, respectively. Thus, we have isomorphisms modulo torsion:

$$
\operatorname{Pic}^{\text {loc }}(0, X) \cong_{\mathbb{Q}} \operatorname{Pic}(S) /\left[\mathcal{O}_{S}(1)\right] \text { and } \operatorname{Pic}^{\text {loc }}(0, \bar{X}) \cong_{\mathbb{Q}} \operatorname{Pic}(\bar{S}) /\left[\mathcal{O}_{\bar{S}}(1)\right] .
$$

More precisely, set $U:=X \backslash\{0\}$ and $\bar{U}:=\bar{X} \backslash\{\overline{0}\}$. Note that $U$ (respectively, $\bar{U}$ ) is a Seifert $\mathbb{C}^{*}$-bundle over $S$ (respectively, $\bar{S}$ ). (See [Kol13b, 9.49-51] for a quick introduction to Seifert bundles in algebraic geometry.) This implies that

$$
H^{2}(\bar{U}, \mathbb{Z}) \cong \mathbb{Z}^{7} \text { and } H^{2}(U, \mathbb{Z}) \cong \mathbb{Z}^{9}
$$

(The Seifert $\mathbb{C}^{*}$-bundle structure gives these modulo torsion. Since both $X$ and $\bar{X}$ are hypersurface singularities, $U$ and $\bar{U}$ are simply connected; thus the above $H^{2}$ are torsion free.) Thus $\pi^{*}$ is not injective on $H^{2}$ and

$$
\mathbb{Z}^{2} \cong H^{1}(E, \mathbb{Z}) \cong \operatorname{ker}\left[H^{2}(U, \mathbb{Z}) \stackrel{\pi^{*}}{\rightarrow} H^{2}(\bar{U}, \mathbb{Z})\right]
$$

By contrast, we claim that $\operatorname{Pic}^{\text {loc }}(0, X)=0$ for very general $g_{4}$. To see this, first note that $\mathbb{Q}$ Cartier divisors $D$ on $S$ correspond to Cartier divisors $\bar{D}$ on $\bar{S}$ for which $\left.\bar{D}\right|_{E}$ is invariant under the Galois involution of $E \rightarrow L$.

Since $\bar{S}$ is a Del Pezzo surface, the pair $(E, \bar{S})$ is obtained from a pair $\left(E, \mathbb{P}^{2}\right)$ by blowing up seven points $P_{1}, \ldots, P_{7} \in E$. Thus the image of $\operatorname{Pic}(\bar{S}) \rightarrow \operatorname{Pic}(E)$ is generated by $\left.\mathcal{O}_{\mathbb{P}^{2}}(1)\right|_{E}$ and the $\mathcal{O}_{E}\left(P_{i}\right)$. For a very general choice of the $P_{i}$, these are independent in $\operatorname{Pic}(E)$ and the only Galois-invariant divisor classes are given by the multiples of $\left.\mathcal{O}_{\mathbb{P}^{2}}(3)\right|_{E} \otimes \mathcal{O}_{E}\left(-P_{1}-\cdots-P_{7}\right)$. This is also the pull-back of the hyperplane class under the projection $S \rightarrow \mathbb{P}_{\mathbf{x}}^{2}$. Therefore $\operatorname{Pic}^{\text {loc }}(0, X)=0$.

\section{Schemes over finite fields}

We start with the following strengthening of Theorem 1.6 over finite fields.

Theorem 6.1. Let $k$ be a finite field and $X$ an excellent $k$-scheme of pure dimension at least 3 that is $S_{2}$ (or at least topologically $S_{2}$; see Definition 6.8). Let $x \in X$ be a point with finite residue field $k(x)$. Let $\pi: \bar{X} \rightarrow X$ denote the normalization and $\bar{x}$ the preimage of $x$. Then the kernel of the pull-back map

$$
\pi^{*}: \operatorname{Pic}^{\mathrm{loc}}(x, X) \rightarrow \operatorname{Pic}^{\mathrm{loc}}(\bar{x}, \bar{X})
$$

is torsion.

Note that, by our Examples 4.5-4.7, the assumptions that $\operatorname{dim} X \geqslant 3, X$ be topologically $S_{2}$ and $k(x)$ be finite are all necessary. 


\section{J. KOLLÁR}

Proof. We factor the normalization $\pi: \bar{X} \rightarrow X$ as

$$
\pi: \bar{X} \stackrel{\pi_{3}}{\longrightarrow} X^{\mathrm{wn}} \stackrel{\pi_{2}}{\longrightarrow} \operatorname{red} X \stackrel{\pi_{1}}{\longrightarrow} X
$$

and show that for each step, the kernel of the pull-back map on the local Picard groups is torsion.

For $\pi_{1}: \operatorname{red} X \rightarrow X$, this is done in Lemma 6.2 , and for $\pi_{2}: X^{\mathrm{wn}} \rightarrow \operatorname{red} X$, this is done in Corollary 6.7. Both of these results are well known and hold in much greater generality. The most delicate part is the finiteness of the kernel for

$$
\pi_{3}^{*}: \operatorname{Pic}^{\text {loc }}\left(x^{\mathrm{wn}}, X^{\mathrm{wn}}\right) \rightarrow \operatorname{Pic}^{\mathrm{loc}}(\bar{x}, \bar{X}) .
$$

This is established in Proposition 6.12, using the quotient theory of [Kol12] and Seifert $\mathbb{G}_{m^{-}}$ bundles as in [Kol13b, 9.53].

Lemma 6.2. Let $k$ be a field of positive characteristic and $X$ a Noetherian $k$-scheme. Then the kernel of the restriction map $\operatorname{Pic}(X) \rightarrow \operatorname{Pic}(\operatorname{red} X)$ is killed by some power of char $k$.

Proof. Let $J \subset \mathcal{O}_{X}$ be an ideal sheaf such that $J^{2}=0$. Set $X_{J}:=\operatorname{Spec} \mathcal{O}_{X} / J$. Note that $j \mapsto 1+j$ identifies $J$ with the kernel of $\mathcal{O}_{X}^{*} \rightarrow \mathcal{O}_{X_{J}}^{*}$. This gives an exact sequence

$$
H^{1}(X, J) \rightarrow H^{1}\left(X, \mathcal{O}_{X}^{*}\right) \rightarrow H^{1}\left(X_{J}, \mathcal{O}_{X_{J}}^{*}\right) .
$$

Since $H^{1}(X, J)$ is a $k$-vector space, it is killed by multiplication by char $k$.

Using this for the powers of the ideal sheaf defining $\operatorname{red} X$, we see that the kernel of the restriction map $\operatorname{Pic}(X) \rightarrow \operatorname{Pic}(\operatorname{red} X)$ is killed by multiplication by a power of char $k$.

The above proof even shows the following stronger result.

Corollary 6.3. Let $k$ be a field of positive characteristic and $(x, X)$ a local Noetherian $k$ scheme that is $S_{2}$ and has pure dimension at least 3. Then the kernel of the restriction map $\mathbf{P i c}^{\mathrm{loc}}(x, X) \rightarrow \mathbf{P i c}^{\mathrm{loc}}(x$, red $X)$ is a connected unipotent group scheme.

Definition 6.4. Let $X$ be a scheme. A partial weak normalization is a finite morphism $\pi: X^{\prime} \rightarrow X$ such that

(i) $X^{\prime}$ is reduced,

(ii) $p$ is a finite, universal homeomorphism and

(iii) the induced morphism $X^{\prime} \rightarrow \operatorname{red} X$ is an isomorphism at all generic points.

The scheme $X$ is called weakly normal if $X$ is reduced and its sole partial weak normalization is the identity $X \cong X$.

If $X$ is excellent, more generally, if the normalization $\bar{X}$ is finite over $X$, then there is a unique maximal partial weak normalization $X^{\text {wn }} \rightarrow X$, called the weak normalization of $X$. Note that $X^{\mathrm{wn}}$ is weakly normal.

If $X$ has residue characteristic 0 , then the weak normalization agrees with the seminormalization. See [Kol96, Section I.7.2] for details.

A weakly normal scheme is $S_{2}$ if and only if it is connected in codimension 1 as in [Har62]. Therefore, if $X$ is $S_{2}$, then so is its weak normalization.

For the proof of the next lemma, see [Kol97, Section 6] or [Kol12, Proposition 35].

Lemma 6.5. Let $k$ be a field of positive characteristic and $g: Y \rightarrow X$ a finite morphism of Noetherian $k$-schemes. The following are equivalent: 


\section{MAPS BetWeEN LOCAL PiCARD GRoups}

(i) The morphism $g$ is a universal homeomorphism.

(ii) The scheme $Y$ is dominated by a Frobenius twist $F_{q}: X_{q} \rightarrow Y \stackrel{g}{\rightarrow} X$ for some power $q$ of char $k$.

Corollary 6.6. Let $k$ be a field of positive characteristic and $g: Y \rightarrow X$ a finite, universal homeomorphism of Noetherian $k$-schemes. Then the kernel of the pull-back map $g^{*}: \operatorname{Pic}(X) \rightarrow$ $\operatorname{Pic}(Y)$ is killed by some power of char $k$.

Proof. By Lemma 6.5, there is a $q=(\operatorname{char} k)^{m}$ such that $F_{q}$ factors as

$$
F_{q}: X_{q} \rightarrow Y \stackrel{g}{\rightarrow} X .
$$

Thus it is enough to prove that the kernel of the pull-back map $F_{q}^{*}: \operatorname{Pic}(X) \rightarrow \operatorname{Pic}\left(X_{q}\right)$ is killed by $q$.

If a line bundle $L$ is given by an open cover $\left\{U_{i}\right\}$ and transition functions $\left\{\phi_{i j}\right\}$, then $F_{q}^{*} L$ can be given by the open cover $\left\{F_{q}^{-1} U_{i}\right\}$ and transition functions $\left\{F_{q}^{*} \phi_{i j}\right\}$.

As an abstract scheme, $X_{q}$ is naturally isomorphic to $X$ and, under this isomorphism, $F_{q}^{*} \phi_{i j}=$ $\phi_{i j}^{q}$. Therefore the Frobenius pull-back $F_{q}^{*} L$ is isomorphic to $L^{\otimes q}$ under this isomorphism. Thus, the kernel of $F_{q}^{*}: \operatorname{Pic}(X) \rightarrow \operatorname{Pic}\left(X_{q}\right)$ is precisely the set of $q$-torsion elements.

Since the weak normalization of an excellent scheme is a finite, universal homeomorphism, as a special case we get the following.

Corollary 6.7. Let $k$ be a field of positive characteristic and $X$ an excellent $k$-scheme with weak normalization $\tau: X^{\mathrm{wn}} \rightarrow X$. Then the kernel of the pull-back map $\operatorname{Pic}(X) \rightarrow \operatorname{Pic}\left(X^{\mathrm{wn}}\right)$ is killed by some power of char $k$.

In contrast with Corollary 6.3, the kernel of $\tau^{*}$ need not be connected, as shown by Example 4.8 .

The last step of the proof uses the concept of topologically $S_{2}$ schemes and the theory of finite, set-theoretic equivalence relations developed in [Kol12].

Definition $6.8\left(S_{2}\right.$ and topologically $\left.S_{2}\right)$. Recall that a scheme $X$ is $S_{2}$ if a finite morphism $g: Y \rightarrow X$ is an isomorphism provided that

(i) there is a closed subset $Z \subset X$ of codimension at least 2 such that $g$ is an isomorphism over $X \backslash Z$ and

(ii) $Y$ has no associated primes supported in $g^{-1}(Z)$.

Similarly, a scheme $X$ is topologically $S_{2}$ if a finite morphism $g: Y \rightarrow X$ is a finite, universal homeomorphism provided that

$\left(1^{\prime}\right)$ there is a closed subset $Z \subset X$ of codimension at least 2 such that $g$ is a finite, universal homeomorphism over $X \backslash Z$ and

$\left(2^{\prime}\right) Y$ has no irreducible components supported in $g^{-1}(Z)$.

It is not hard to see that a pure-dimensional scheme $X$ is topologically $S_{2}$ if and only if the following holds:

$\left(3^{\prime}\right)$ Let $\pi: U \rightarrow X$ be an étale morphism from a connected scheme $U$ and $Z \subset U$ a closed subscheme of codimension at least 2 . Then $U \backslash Z$ is connected. 


\section{J. KOLLÁR}

These imply that an $S_{2}$ scheme is topologically $S_{2}$, a weakly normal scheme is $S_{2}$ if and only if it is topologically $S_{2}$, and the weak normalization of an $S_{2}$ scheme is topologically $S_{2}$. We also use that by [Gro68, XIII.2.1] a Cartier divisor in a topologically $S_{2}$ scheme is also topologically $S_{2}$.

6.9 (Set-theoretic equivalence relations). (For more details, see [Kol12].)

Let $X$ be an excellent scheme, $\pi: \bar{X} \rightarrow X$ its normalization and $R$ the normalization of $\bar{X} \times_{X} \bar{X}$. Together with the coordinate projections $\sigma_{1}, \sigma_{2}: R \rightrightarrows \bar{X}$ we have a finite, set-theoretic equivalence relation.

Let $Q \subset R$ be a closed subscheme such that $\left.\sigma_{1}\right|_{Q},\left.\sigma_{2}\right|_{Q}: Q \rightrightarrows \bar{X}$ is also a set-theoretic equivalence relation. By [Kol12, Lemma 1.7], the geometric quotient $\bar{X} / Q$ exists and the geometric fibers of $\bar{X} \rightarrow \bar{X} / Q$ are precisely the $Q$-equivalence classes.

In particular, if $Q=R$, then $\bar{X} / R \rightarrow X$ is a finite, universal homeomorphism. If $X$ is weakly normal, then $\bar{X} / R=X$.

Finally, we study what happens as we go from a weakly normal scheme to its normalization.

Proposition 6.10. Let $k$ be a field of characteristic $p>0$ and $X$ an excellent $k$-scheme of pure dimension at least 3 that is weakly normal and $S_{2}$. Let $\pi: \bar{X} \rightarrow X$ denote the normalization. Let $x \in X$ be a closed point with residue field $k(x)$. Then there are a $k$-torus $\mathbb{T}$ and a linear representation

$$
I_{x}: \operatorname{ker}\left[\mathbf{P i c}^{\mathrm{loc}}(x, X) \stackrel{\pi^{*}}{\rightarrow} \mathbf{P i c}^{\mathrm{loc}}(\bar{x}, \bar{X})\right] \rightarrow \mathbb{T}
$$

whose kernel is $p^{\infty}$-torsion.

Proof. Let $L$ be a line bundle on $X \backslash\{x\}$ such that $\pi^{*} L$ is trivial on $\bar{X} \backslash\{\bar{x}\}$.

We can view $L$ as a $\mathbb{G}_{m}$-bundle over $X \backslash\{x\}$; thus $\pi^{*} L$ is a trivial $\mathbb{G}_{m}$-bundle over $\bar{X} \backslash\{\bar{x}\}$. It extends to a trivial $\mathbb{G}_{m}$-bundle $\bar{L}$ over $\bar{X}$. Since $X$ is excellent, $\bar{X}$ is finite over $X$; hence $\bar{L}$ is of finite type over $X$.

Let $R$ be the normalization of $\bar{X} \times_{X} \bar{X}$. Then $\sigma_{1}, \sigma_{2}: R \rightrightarrows \bar{X}$ is a set-theoretic finite equivalence relation. Since $X$ is weakly normal, the geometric quotient $\bar{X} / R$ equals $X$; see $\S 6.9$.

Let $r \subset R$ denote the union of the reduced preimages of $\bar{x}$. Since $\pi^{*} L$ is pulled back from $X$, we have isomorphisms

$$
\phi_{R \backslash r}: \sigma_{1}^{*}\left(\pi^{*} L\right) \cong \sigma_{2}^{*}\left(\pi^{*} L\right)
$$

over $R \backslash r$. By assumption $\pi^{*} L$ is trivial, thus $\phi_{R \backslash r}$ can be viewed as an isomorphism of two trivial $\mathbb{G}_{m}$-bundles on $R \backslash r$.

Let $R^{[1]} \subset R$ be the union of the irreducible components of dimension at least $\operatorname{dim} X-1$ and $R^{(1)} \subset R$ the equivalence relation generated by $R^{[1]}$. We will check at the end that $\bar{X} / R=$ $\bar{X} / R^{(1)}$.

Since $R^{[1]}$ is normal and every irreducible component has dimension at least 2 , the isomorphism $\phi_{R \backslash r}$ extends to an isomorphism

$$
\phi_{R}^{[1]}: \sigma_{1}^{*}(\bar{L}) \cong \sigma_{2}^{*}(\bar{L})
$$

over $R^{[1]}$. The isomorphisms (6.10.2) define a $\mathbb{G}_{m}$-equivariant extension of the relation $R^{(1)}$ to a finite relation on $\bar{L}$. Let $\bar{R}_{L}$ denote the equivalence relation generated by this extension. In general, such an extension is a profinite equivalence relation.

In our case the only nonfiniteness can occur over $x \in X$. For $\bar{x}_{i} \in \bar{x}$, let $\mathbb{G}_{m}\left(\bar{x}_{i}\right)$ denote the multiplicative group scheme of the residue field $k\left(\bar{x}_{i}\right)$. For a point $r_{\ell} \in R^{(1)}$ lying over $x$, set 


\section{MAPS BeTWEen LOCAL PiCARD GROUPS}

$\bar{x}_{i}:=\sigma_{1}\left(r_{\ell}\right)$ and $\bar{x}_{j}:=\sigma_{2}\left(r_{\ell}\right)$. (We allow $i=j$.) Then $\phi_{R}^{[1]}$ gives an isomorphism

$$
\phi_{i j \ell}: \mathbb{G}_{m}\left(\bar{x}_{i}\right) \cong \mathbb{G}_{m}\left(\bar{x}_{j}\right)
$$

that is defined over $k\left(r_{\ell}\right)$.

It is enough to prove the theorem after a finite field extension. We can thus replace $k$ by the composition of the above $k\left(\bar{x}_{i}\right)$ and $k\left(r_{\ell}\right)$.

Let $\Gamma_{x}$ denote the graph whose vertices are the points $\bar{x}_{i}$. To each $r_{\ell}$ we add an edge connecting $\bar{x}_{i}$ and $\bar{x}_{j}$. After we fix a base point $\bar{x}_{0}$, compositions of the above $\phi_{i j \ell}$ define a homomorphism

$$
I_{x}(L): H_{1}\left(\Gamma_{x}, \mathbb{Z}\right) \rightarrow \mathbb{G}_{m}(k) .
$$

The construction is compatible with field extensions and Henselization, thus we get a homomorphism

$$
I_{x}: \operatorname{ker}\left[\mathbf{P i c}^{\mathrm{loc}}(x, X) \stackrel{\pi^{*}}{\longrightarrow} \mathbf{P i c}^{\mathrm{loc}}(\bar{x}, \bar{X})\right] \rightarrow \operatorname{Hom}\left(H_{1}\left(\Gamma_{x}, \mathbb{Z}\right), \mathbb{G}_{m}\right) .
$$

Next, assume that $L$ is in the kernel of $I_{x}$. Then $\bar{R}_{L}$ is a finite equivalence relation. More precisely, each $\bar{R}_{L}$-equivalence class over $x$ contains exactly one point of $\mathbb{G}_{m}\left(\bar{x}_{0}\right)$.

Since $\bar{L}$ is of finite type over $X$, by [Kol12, Theorem 6] the geometric quotient $\bar{L} / \bar{R}_{L}$ exists. It is a Seifert $\mathbb{G}_{m}$-bundle over $\bar{X} / R^{(1)}$ by [Kol13b, 9.48]. (The statement there assumes that two other conditions (HN) and (HSN) are also satisfied. These are, however, used only to ensure that the geometric quotient $\bar{L} / \bar{R}_{L}$ exists. In our case existence is assured by [Kol12, Theorem 6], the rest of the proof then works.) By [Kol13b, 9.53], a power $\bar{L}^{\otimes q}$ of $\bar{L}$ descends to a $\mathbb{G}_{m}$-bundle over $\bar{X} / R^{(1)}$ for some $q=p^{r}$.

Finally, let $R^{(2)} \subset R$ denote the union of all irreducible components of dimension at most $\operatorname{dim} X-2$. Let $Z \subset X$ be the image of $R^{(2)}$. Then $Z$ has codimension at least 2 in $X$ and $R$ agrees with $R^{(1)}$ over $X \backslash Z$. Thus $\bar{X} / R^{(1)} \rightarrow X$ is a finite, universal homeomorphism over $X \backslash Z$. Since $X$ is $S_{2}$, this implies that $\bar{X} / R^{(1)} \rightarrow X$ is a finite, universal homeomorphism over $X$. By construction, it is an isomorphism at all generic points. Since $X$ is weakly normal, this implies that $\bar{X} / R^{(1)}=X$. Thus $L^{\otimes m}$ extends to a $\mathbb{G}_{m}$-bundle over $X$, hence it is trivial in $\operatorname{Pic}^{\text {loc }}(x, X)$.

6.11. Note that we have proved that $I_{x}$ is a homomorphism of algebraic groups, but the proof did not establish that its image is a closed algebraic subgroup of $\mathbb{T}$. (For instance we did not exclude the possibility that $\operatorname{ker} \pi^{*} \cong \mathbb{Z}$ and $I_{x}$ is an injection.)

However, once we know that ker $\pi^{*}$ in Theorem 1.6 is of finite type, Proposition 6.10 implies that $\operatorname{ker} \pi^{*}$ is in fact linear.

Corollary 6.12. Let $k$ be a finite field and $X$ an excellent $k$-scheme of pure dimension at least 3 that is weakly normal and $S_{2}$. Let $x \in X$ be a closed point. Then the kernel of the pull-back map

is torsion.

$$
\pi^{*}: \operatorname{Pic}^{\mathrm{loc}}(x, X) \rightarrow \operatorname{Pic}^{\mathrm{loc}}(\bar{x}, \bar{X})
$$

In contrast with the previous steps, the order of the torsion kernel need not be a power of char $k$.

Proof. Let $L$ be a line bundle on $X \backslash\{x\}$ such that $\pi^{*} L$ is trivial on $\bar{X} \backslash\{\bar{x}\}$. In (6.10.4) we constructed a homomorphism

$$
I_{x}(L): H_{1}\left(\Gamma_{x}, \mathbb{Z}\right) \rightarrow \mathbb{G}_{m}(k) .
$$




\section{J. KOLLÁR}

If $k$ is finite, then $\mathbb{G}_{m}(k)$ is a torsion group. Thus $I_{x}\left(L^{m}\right)$ is the trivial homomorphism for some $m>0$. By Proposition 6.10 this implies that $L^{m}$ is $p^{\infty}$-torsion; hence $L$ is a torsion element of $\operatorname{Pic}^{\text {loc }}(x, X)$.

We can now prove the following form of Theorem 1.4 over finite fields.

Theorem 6.13. Let $k$ be a finite field, $X$ and $Y$ excellent $k$-schemes of pure dimension at least 3 that are topologically $S_{2}$ and $\pi: Y \rightarrow X$ a finite morphism. Let $x \subset \pi(Y) \subset X$ be a finite subscheme and $y \subset Y$ the preimage of $x$.

Assume that $\pi(Y)$ contains the support of a complete intersection subscheme $x \subset Z \subset X$ of dimension at least 3 .

Then the kernel of the natural map $\operatorname{Pic}^{\text {loc }}(x, X) \rightarrow \operatorname{Pic}^{\text {loc }}(y, Y)$ consists of torsion elements.

Proof. We use induction on the dimension of $X$. If $\operatorname{dim} X \leqslant 3$, then in fact $X=Z$ and so $\pi$ is a finite surjection. This case is treated in Theorem 6.1 and $\S 2.4$.

Thus, assume $\operatorname{dim} X \geqslant 4$. If $\tau: Y^{\prime} \rightarrow Y$ is a finite morphism and $Z \subset(\pi \circ \tau)\left(Y^{\prime}\right)$, then the kernel of $\operatorname{Pic}^{\text {loc }}(x, X) \rightarrow \operatorname{Pic}^{\text {loc }}\left(y^{\prime}, Y^{\prime}\right)$ contains the kernel of $\operatorname{Pic}^{\text {loc }}(x, X) \rightarrow \operatorname{Pic}^{\operatorname{loc}}(y, Y)$. Thus we may assume that $Y \rightarrow X$ factors through the normalization $\bar{X} \rightarrow X$, that $\pi(Y)=\operatorname{Supp} Z$ and that $Y$ is normal. Using Theorem 6.1 we may thus also assume that $X$ is normal.

Since $Z \subset X$ is a complete intersection, it is also a complete intersection inside some Cartier divisor $D \subset X$. As we noted in Definition 6.8, the divisor $D$ is also topologically $S_{2}$ by [Gro68, XIII.2.1]. Using [BdJ14], we can thus replace $X$ by $D$ and finish by induction.

\section{Using the relative Picard group}

Theorems 1.4, 1.6 and 1.7 assert that certain line bundles are contained in $\mathbf{P i c}^{\operatorname{loc}-\tau}(x, X)$. We plan to prove such results by first establishing the claim over finite fields and then going back to arbitrary fields. As we already noted in Question 2.1, we need a method to decide when a line bundle on $X \backslash\{x\}$ is contained in $\mathbf{P i c}^{\operatorname{loc}-\tau}(x, X)$.

7.1 (General set-up). Theorems 1.4, 1.6 and 1.7 can be formulated as follows.

Step 0: Starting point. Let $p:(y, Y) \rightarrow(x, X)$ be a finite morphism of local $k$-schemes and $L \in \operatorname{Pic}^{\text {loc }}(x, X)$ such that $\pi^{*} L$ is in $\mathbf{P i c}^{\operatorname{loc}-\tau}(y, Y)$. By passing to a power of $L$, we may assume that $\pi^{*} L$ is in $\mathbf{P i c}^{\text {loc-o}}(y, Y)$, that is, $\pi^{*} L$ is algebraically equivalent to the trivial bundle.

We would like to prove that, under suitable conditions, $L \in \mathbf{P i c}^{\operatorname{loc}-\tau}(x, X)$.

Step 1: Spreading out. There is an integral $\mathbb{Z}$-scheme of finite type $S$ such that we have the following.

(i) There are $S$-schemes of finite type $Y_{S} \rightarrow S$ and $X_{S} \rightarrow S$ with sections $\sigma_{Y}: S \rightarrow Y_{S}$ and $\sigma_{X}: S \rightarrow X_{S}$

(ii) There is a finite morphism $p_{S}: Y_{S} \rightarrow X_{S}$ such that $\sigma_{X}=p_{S} \circ \sigma_{Y}$ and $\operatorname{red} p_{S}^{-1}\left(\sigma_{X}(S)\right)=$ $\sigma_{Y}(S)$.

(iii) There is a line bundle $L_{S}$ on $X_{S} \backslash \sigma_{X}(S)$ such that for every $s \in S$, the restriction $\left.p_{S}^{*} L_{S}\right|_{Y_{s}}$ is algebraically equivalent to the trivial bundle on $Y_{s}$.

(iv) There is a map to the generic point $\operatorname{Spec} k \rightarrow S$ such that

$$
(p: Y \rightarrow X, L) \cong \operatorname{Spec} k \times_{S}\left(p_{S}: Y_{S} \rightarrow X_{S}, L_{S}\right)
$$




\section{MAPS BetWeEN LOCAL PiCARD GRoups}

There are several known results that say that certain good properties of the generic fiber are inherited by all fibers, at least over a dense open subset; see [Gro60, IV.12] for long lists. For example, if the generic fiber is $S_{2}$ and has pure dimension $d$, then, possibly after shrinking $S$, we may assume that every fiber is $S_{2}$ and has pure dimension $d$.

Step 2: Over finite fields. For every closed point $s \in S$ we have a finite morphism $p_{s}:\left(y_{s}, Y_{s}\right)$ $\rightarrow\left(x_{s}, X_{s}\right)$ and an $L_{s} \in \mathrm{Pic}^{\text {loc }}\left(x_{s}, X_{s}\right)$ such that $p_{s}^{*} L_{s}$ is algebraically equivalent to the trivial bundle.

This is an instance of the original problem over the residue field $k(s)$. Since $S$ is a $\mathbb{Z}$-scheme of finite type, these residue fields are all finite. Thus Theorem 6.13 implies that, under our assumptions, $L_{s}^{m_{s}}$ is trivial for some $m_{s}>0$.

If $m_{s}=m$ is independent of $s$, then it is reasonable to expect that $L^{m} \cong \mathcal{O}_{X}$. However, this is usually not the case. If $\mathbf{P i c}{ }^{\text {loc-o}}(x, X)$ has positive dimension and char $k=0$, then there are nontorsion line bundles $L \in \mathbf{P i c}^{\text {loc-o }}(x, X)$. For these, the $m_{s}$ are not even bounded.

Step 3: Lifting: easy case. If $\operatorname{depth}_{x_{s}} X_{s} \geqslant 3$, then [Gro68, XI.3.16] implies that $L^{m_{s}} \cong \mathcal{O}_{X}$ and we are done. This happens precisely when $\mathbf{P i c}^{\text {loc-o}}(x, X) \cong \operatorname{Spec} k$; thus $\mathbf{P i c}^{\text {loc- }-\tau}(x, X)$ is identified with the torsion subgroup of $\operatorname{NS}^{\text {loc }}(x, X)$.

Step 4: Lifting: hard case. At this point we can forget about $Y$ and $\pi: Y \rightarrow X$ in the original set-up. Thus from now on we have only the following data:

(i) a local $k$-scheme $(x, X)$ and an $L \in \operatorname{Pic}^{\text {loc }}(x, X)$

(ii) a finitely generated $\mathbb{Z}$-subalgebra $A \subset k$ and its spectrum $S$

(iii) an $S$-scheme of finite type $X_{S} \rightarrow S$ with a section $\sigma_{X}: S \rightarrow X_{S}$ (we set $x_{s}:=\sigma_{X}(s)$ for $s \in S)$

(iv) a line bundle $L_{S}$ on $X_{S} \backslash \sigma_{X}(S)$ such that $L_{s} \in \operatorname{Pic}^{\text {loc }}\left(x_{s}, X_{s}\right)$ is torsion for every closed point $s \in S$

(v) an isomorphism $(x, X, L) \cong \operatorname{Spec} k \times_{S}\left(\sigma_{X}(S), X_{S}, L_{S}\right)$

The proofs of Theorems 1.4, 1.6 and 1.7 will be completed by the next result.

Theorem 7.2. Let $S$ be an integral scheme whose closed points are dense in $S$. Let $f: X \rightarrow S$ be a flat morphism whose fibers are $S_{2}$ and have pure dimension at least 3. Let $\sigma: S \rightarrow X$ be a section and $L$ a line bundle on $X \backslash \sigma(S)$.

Assume that for a dense set of closed point $s \in S$ there is a natural number $m_{s} \in \mathbb{N}$ such that $L_{s}^{m_{s}} \cong \mathcal{O}_{U_{s}}$, where $U_{s}:=X_{s} \backslash\left\{x_{s}\right\}$.

Then $L_{k(S)} \in \mathbf{P i c}^{\text {loc }-\tau}\left(x_{k(S)}, X_{k(S)}\right)$.

Remark 7.3. It is possible that the assumption of closed points being dense is not necessary. By Proposition 8.5 this holds if the universal deformation in Proposition 8.2 admits an algebraization. I am able to prove only a weaker version of this: an algebraization of Pic ${ }^{\text {loc }-\circ}$ over the generic fiber gives an algebraization of the universal deformation over an open subset of $S$. This is why we need to assume that closed points are dense in $S$, an assumption that always holds in our applications.

\section{Formal deformation theory of Pic ${ }^{\text {loc }}$}

8.1. Let $(s, S)$ be a local scheme, $(y, Y)$ a local Henselian scheme and $g: Y \rightarrow S$ a flat, affine morphism. 


\section{J. KOLLÁR}

Fix a line bundle $L$ on $Y_{s} \backslash\{y\}$. Let $\left(A, m_{A}\right)$ be a local Artin $\mathcal{O}_{S}$-algebra with residue field $k=A / m_{A}$. Set $Y_{A}:=Y \times_{S} \operatorname{Spec} A$ and $U_{A}:=Y_{A} \backslash\{y\}$.

Let $\operatorname{Def}_{S}(L, A)$ denote the set of isomorphism classes of line bundles on $U_{A}$ whose restriction to $U_{k}$ is isomorphic to (the pull-back of) $L$.

We check the conditions of [Sch68] for the prorepresentability of the functor $A \mapsto \operatorname{Def}_{S}(L, A)$. Consider an extension

$$
0 \rightarrow M \rightarrow B \rightarrow A \rightarrow 0
$$

such that $M^{2}=0$. Correspondingly, there is an exact sequence

$$
0 \rightarrow M \otimes \mathcal{O}_{U_{s}} \stackrel{m \mapsto 1+m}{\longrightarrow} \mathcal{O}_{U_{B}}^{*} \rightarrow \mathcal{O}_{U_{A}}^{*} \rightarrow 1
$$

Pick any $\gamma_{A} \in H^{0}\left(U_{A}, \mathcal{O}_{U_{A}}^{*}\right)$. If the fibers of $f$ are $S_{2}$, then $\gamma_{A}$ extends to a section $\gamma_{A}^{\prime} \in$ $H^{0}\left(Y_{A}, \mathcal{O}_{Y_{A}}\right)$. Since $f$ is affine, we can lift $\gamma_{A}^{\prime}$ to $\gamma_{B}^{\prime} \in H^{0}\left(Y_{B}, \mathcal{O}_{Y_{B}}\right)$ and then restrict to $\gamma_{B} \in$ $H^{0}\left(U_{B}, \mathcal{O}_{U_{B}}^{*}\right)$. Thus

$$
H^{0}\left(U_{B}, \mathcal{O}_{U_{B}}^{*}\right) \rightarrow H^{0}\left(U_{A}, \mathcal{O}_{U_{A}}^{*}\right)
$$

is surjective. Thus the following is a piece of the long exact cohomology sequence of (8.1.2):

$$
0 \rightarrow M \otimes H^{1}\left(U_{s}, \mathcal{O}_{U_{s}}\right) \rightarrow H^{1}\left(U_{B}, \mathcal{O}_{U_{B}}^{*}\right) \rightarrow H^{1}\left(U_{A}, \mathcal{O}_{U_{A}}^{*}\right) \stackrel{\text { obs }}{\longrightarrow} M \otimes H^{2}\left(U_{s}, \mathcal{O}_{U_{s}}\right),
$$

where obs is called the obstruction map. If (8.1.1) splits, then the liftings form a principal homogeneous space under $M \otimes H^{1}\left(U_{s}, \mathcal{O}_{U_{s}}\right)$; the latter is independent of $A$. Furthermore, if $\operatorname{dim} Y_{s} \geqslant 3$, then $H^{1}\left(U_{s}, \mathcal{O}_{U_{s}}\right)$ is finite dimensional by [Gro68, VIII.2.3].

It remains to understand liftings to $D:=B \times{ }_{A} C$, where $B \rightarrow A$ and $C \rightarrow A$ are maps of Artin $\mathcal{O}_{S}$-algebras. A line bundle $L_{D}$ on $U_{D}$ is determined by a line bundle $L_{B}$ on $U_{B}$, a line bundle $L_{C}$ on $U_{C}$ plus an isomorphism $\phi:\left.\left.L_{C}\right|_{U_{A}} \cong L_{B}\right|_{U_{A}}$. Any different isomorphism is given by $\gamma_{A} \cdot \phi$, where $\gamma_{A} \in H^{0}\left(U_{A}, \mathcal{O}_{U_{A}}^{*}\right)$. By (8.1.3) one can lift $\gamma_{A}$ to $\gamma_{B} \in H^{0}\left(U_{B}, \mathcal{O}_{U_{B}}^{*}\right)$. Thus instead of changing $\phi$ by $\gamma_{A}$, we can change $L_{B}$ by the isomorphism $\gamma_{B}: L_{B} \rightarrow L_{B}$ to conclude that $L_{D}$ does not depend on $\phi$. That is,

$$
\operatorname{Def}_{S}\left(L, B \times{ }_{A} C\right) \cong \operatorname{Def}_{S}(L, B) \times \operatorname{Def}_{S}(L, A) \operatorname{Def}_{S}(L, C) .
$$

We have thus proved the following.

Proposition 8.2. Let $(s, S)$ be a local scheme, $(y, Y)$ a local Henselian scheme and $g: Y \rightarrow S$ a flat morphism with $S_{2}$ fibers of dimension at least 3. Let $L$ be a line bundle on $Y_{s} \backslash\{y\}$. Then $A \mapsto \operatorname{Def}_{S}(L, A)$ is prorepresentable by a complete, local, Noetherian $\mathcal{O}_{S}$-algebra $\operatorname{Def}_{S}(L)$.

Definition 8.3. Let $S$ be a (nonlocal) scheme and $f: X \rightarrow S$ a flat morphism with $S_{2}$ fibers of pure dimension at least 3. Let $\sigma: S \rightarrow X$ be a section. For each $s \in S$, we are especially interested in two deformation functors.

(8.3.1) $\operatorname{Def}_{(s, S)}\left(\mathcal{O}_{X_{s}}\right)$ is obtained by applying Proposition 8.2 to the localization $(s, S)$ of $S$ at $s$ and the Henselization $(y, Y)$ of $X$ at $\sigma(s)$. This is an $\mathcal{O}_{s, S}$-algebra parametrizing deformations (over Artin algebras over $\mathcal{O}_{s, S}$ ) of the trivial line bundle $\mathcal{O}_{X_{s}}$ (pulled back to $Y_{s} \backslash\{y\}$ ).

(8.3.2) $\operatorname{Def}_{k(s)}\left(\mathcal{O}_{X_{s}}\right)$ is obtained by applying Proposition 8.2 to $S:=\{s\}$ and the Henselization $(y, Y)$ of the fiber $X_{s}$ at $\sigma(s)$. This is a $k(s)$-algebra parametrizing deformations (over Artin algebras over the residue field $k(s)$ ) of the trivial line bundle $\mathcal{O}_{X_{s}}$ (pulled back to $Y_{s} \backslash\{y\}$ ).

Note that

$$
\operatorname{Def}_{k(s)}\left(\mathcal{O}_{X_{s}}\right) \cong \operatorname{Def}_{(s, S)}\left(\mathcal{O}_{X_{s}}\right) \otimes_{S} k(s)
$$




\section{MAPS BeTWEen LOCAL PiCARD GROUPS}

Definition 8.4 (Universal families). Let $S$ be a scheme, $f: X \rightarrow S$ a flat morphism with $S_{2}$ fibers and $\sigma: S \rightarrow X$ a section. A family in $P i c^{\text {loc }}$ over $P$ is given by

(i) a morphism $p: P \rightarrow S$,

(ii) an étale morphism $g: Y \rightarrow X \times{ }_{S} P$,

(iii) a lifting of the closed subscheme $\left(\sigma, 1_{P}\right): P=S \times_{S} P \hookrightarrow X \times{ }_{S} P$ to $\sigma_{P}: P \rightarrow Y$ and

(iv) a line bundle $L_{Y}$ on $Y \backslash \sigma_{P}(P)$.

For a point $z \in P$, the corresponding line bundle on $Y_{p(z)} \backslash \sigma_{P}(z)$ is denoted by $L_{z}$.

These data give a finite-type family in $P i c^{\text {loc }}$ if $P$ is of finite type over $S$.

The above family is a deformation of the trivial bundle if, in addition,

(v) there is a section $\rho: S \rightarrow P$ such that the restriction of $L_{Y}$ to $Y_{\rho} \backslash \sigma_{P}(P)$ is isomorphic to the structure sheaf, where $Y_{\rho}:=g^{-1}\left(X \times_{S} \rho(S)\right)$.

For every $s \in S$ we can localize at $s$ and get a deformation of $\mathcal{O}_{Y_{s}}$ as in (8.1).

We say that the above family is universal at a line bundle $L_{s}$ on $X_{s} \backslash \sigma(s)$ corresponding to a point $z \in P_{s}$ if

(vi) the induced map

$$
\operatorname{Def}_{(s, S)}\left(L_{s}\right) \rightarrow \widehat{\mathcal{O}}_{z, P}
$$

is an isomorphism.

It is probably not necessary, but for technical reasons it is easier to insist that a universal family satisfy the following:

(vii) The morphism $\operatorname{red} p: \operatorname{red} P \rightarrow \operatorname{red} S$ is smooth.

This always holds in characteristic 0 . In positive characteristic it can be achieved after a purely inseparable, dominant (but not necessarily finite) base change $S^{\prime} \rightarrow S$ and by passing to an open subset $P^{\prime} \subset P$.

Proposition 8.5. Let $(s, S)$ be an integral, local scheme with function field $K$. Let $f: X \rightarrow S$ be a flat morphism with $S_{2}$ fibers, $\sigma: S \rightarrow X$ a section and $L$ a line bundle on $X \backslash \sigma(S)$. Assume that

(i) there is a finite type family as in Definition 8.4(1)-(7) that is universal at $\mathcal{O}_{X_{s}}$ and

(ii) $L_{s} \cong \mathcal{O}_{X_{s}}$.

Then $L_{K} \in \mathbf{P i c}^{\text {loc-o}}\left(x_{K}, X_{K}\right)$.

Proof. For every Artin $\mathcal{O}_{s, S}$-algebra $A$, the line bundle $L$ defines a deformation of $\mathcal{O}_{X_{s}}$ over $A$ since $L_{s} \cong \mathcal{O}_{X_{s}}$. This gives a formal deformation of $L_{s} \cong \mathcal{O}_{X_{s}}$ over $\hat{S}$, the completion of $S$ at $s$.

By assumption $\operatorname{Def}_{(s, S)}\left(\mathcal{O}_{X_{s}}\right) \cong \widehat{\mathcal{O}}_{\rho(s), P}$, thus there is a section $u: \widehat{S} \rightarrow P$ such that $\left.\left(u^{*} L_{Y}\right)\right|_{X_{A}}$ is isomorphic to $\left.L\right|_{X_{A}}$ for every Artin $\mathcal{O}_{s, S}$-algebra $A$. Proposition 8.6 then implies that $\left.u^{*} L_{Y} \cong L\right|_{\hat{S}}$. Since $S$ is integral, the section $u$ factors through $\operatorname{red} P$.

Now we use that red $: \operatorname{red} P \rightarrow \operatorname{red} S$ is smooth (Definition 8.4(7)). Thus $\widehat{\mathcal{O}}_{\rho(s) \text {,red } P}$ is a formal power series ring over $\widehat{\mathcal{O}}_{s, S}$ and so each formal section of red $p$ can be given by an ideal $\left(t_{1}-\right.$ $\left.g_{1}, \ldots, t_{m}-g_{m}\right)$, where $g_{i} \in \widehat{\mathcal{O}}_{s, S}$. Thus any two sections are algebraically equivalent. This implies that $L_{K}$ and $\mathcal{O}_{X_{K}}$ are algebraically equivalent over $k(\hat{S})$, hence also over $K$ by (3.3.3). 


\section{J. KOLLÁR}

Proposition 8.6. Let $(s, S)$ be a local scheme with maximal ideal $m$. Let $f: X \rightarrow S$ be a scheme, flat over $S$ with $S_{2}$-fibers. Let $X_{n}:=\operatorname{Spec}_{X} \mathcal{O}_{X} / m^{n+1} \mathcal{O}_{X}$ be the $n$th infinitesimal neighborhood of $X_{0}:=X_{s}$. Let $Z \subset X$ be a subscheme that is finite over $S$ and $j: X \backslash Z \hookrightarrow X$ and $j_{n}: X_{n} \backslash Z_{n} \hookrightarrow$ $X_{n}$ the natural injections. Let $L$ be an invertible sheaf on $X \backslash Z$ and $L_{n}:=L_{X_{n} \backslash Z_{0}}$. Assume that one of the following holds:

(i) The line bundle $\left(j_{n}\right)_{*}\left(L_{n}\right)$ is locally free for every $n \geqslant 0$.

(ii) The line bundle $\left(j_{0}\right)_{*}\left(L_{0}\right)$ is locally free and $R^{1}\left(j_{0}\right)_{*}\left(L_{0}\right)=0$.

Then $j_{*} L$ is invertible in a neighborhood of $Z_{0}$.

Proof. We may assume that $\mathcal{O}_{S}$ is $m$-adically complete and, possibly after passing to a smaller neighborhood of $Z_{0}$, we may assume that $f$ is affine and $\left(j_{0}\right)_{*}\left(L_{0}\right) \cong \mathcal{O}_{X_{0}}$. For every $n$ we have an exact sequence

$$
0 \rightarrow\left(m_{0}^{n} / m_{0}^{n+1}\right) \otimes L_{0} \rightarrow L_{n} \rightarrow L_{n-1} \rightarrow 0
$$

Pushing it forward, we get an exact sequence

$$
\begin{aligned}
0 & \rightarrow\left(m_{0}^{n} / m_{0}^{n+1}\right) \otimes\left(j_{0}\right)_{*}\left(L_{0}\right) \rightarrow\left(j_{n}\right)_{*}\left(L_{n}\right) \stackrel{r_{n}}{\rightarrow}\left(j_{n-1}\right)_{*}\left(L_{n-1}\right) \rightarrow \\
& \rightarrow\left(m_{0}^{n} / m_{0}^{n+1}\right) \otimes R^{1}\left(j_{0}\right)_{*}\left(L_{0}\right) .
\end{aligned}
$$

If $\left(j_{n}\right)_{*}\left(L_{n}\right)$ is locally free, then so is its restriction to $X_{n-1}$, and $r_{n}$ gives a map of locally free sheaves

$$
\bar{r}_{n}:\left.\left(j_{n}\right)_{*}\left(L_{n}\right)\right|_{X_{n-1}} \rightarrow\left(j_{n-1}\right)_{*}\left(L_{n-1}\right)
$$

that is an isomorphism on $X_{n-1} \backslash Z_{n-1}$. Since $\operatorname{depth}_{Z_{n-1}} X_{n-1} \geqslant 2$, this implies that $\bar{r}_{n}$ is an isomorphism and so $r_{n}$ is surjective. The vanishing of $R^{1}\left(j_{0}\right)_{*}\left(L_{0}\right)$ also implies that $r_{n}$ is surjective. Thus each $\left(j_{n}\right)_{*}\left(L_{n}\right)$ is locally free along $X_{n}$, and the constant 1 section of $\left(j_{0}\right)_{*}\left(L_{0}\right) \cong$ $\mathcal{O}_{X_{0}}$ lifts back to a nowhere zero global section of $\lim _{\longleftarrow}\left(j_{n}\right)_{*}\left(L_{n}\right)$. Hence $\varliminf_{\lim }\left(j_{n}\right)_{*}\left(L_{n}\right) \cong \mathcal{O}_{X}$.

Furthermore, we have a natural map $j_{*} L \rightarrow \lim \left(j_{n}\right)_{*}\left(L_{n}\right) \cong \mathcal{O}_{X}$ that is an isomorphism on $X \backslash Z$. Since $\operatorname{depth}_{Z} j_{*} L \geqslant 2$, this implies that $\overleftarrow{j_{*} L} \cong \mathcal{O}_{X}$.

The examples below show that going from formal triviality of deformations to actual triviality is not automatic.

Example 8.7. Let $(e, E) \cong\left(e, E^{\prime}\right)$ be an elliptic curve. Set $X:=(E \backslash\{e\}) \times E^{\prime}$, and let $p: X \rightarrow E^{\prime}$ be the second projection. Let $\Delta \subset X$ be the diagonal and $L=\mathcal{O}_{X}(\Delta)$.

For $p \in E^{\prime} \backslash\{e\}$, the line bundle $\left.L\right|_{X_{p}}$ is a nontrivial element of

$$
\operatorname{Pic}\left(X_{p} \backslash\{e\}\right) \cong \operatorname{Pic}(E \backslash\{e\}) \cong \mathbf{P i c}^{\circ}(E),
$$

but $\left.L\right|_{X_{e}}$ is trivial.

For $m \in \mathbb{N}$, let $X_{m} \subset X$ denote the $m$ th infinitesimal thickening of the fiber $X_{1}:=X_{e}$. We have exact sequences

$$
H^{1}\left(X_{1}, \mathcal{O}_{X_{1}}\right) \rightarrow H^{1}\left(X_{m+1}, \mathcal{O}_{X_{m+1}}^{*}\right) \rightarrow H^{1}\left(X_{m}, \mathcal{O}_{X_{m}}^{*}\right) \rightarrow H^{2}\left(X_{1}, \mathcal{O}_{X_{1}}\right) .
$$

Since $X_{1} \cong E \backslash\{e\}$ is affine, this shows that

$$
\operatorname{Pic}\left(X_{m} \backslash\{e\}\right) \cong \operatorname{Pic}(E \backslash\{e\}) \cong \operatorname{Pic}^{\circ}(E) .
$$

Thus $\left.L\right|_{X_{m}}$ is trivial for every $m$. 


\section{Maps Between LOCAL PicARd Groups}

Example 8.8. Consider the family of smooth, affine surfaces

$$
S:=\left(x^{2}+y^{2}=1+t^{2} z^{2}\right) \subset \mathbb{A}_{x y z}^{3} \times \mathbb{A}_{t}^{1} .
$$

Set $D:=(x-1=y-t z=0)$ and $L:=\mathcal{O}_{S}(D)$.

The surface $S_{t}$ is a hyperboloid for $t \neq 0$; thus $\operatorname{Pic}\left(S_{t}\right) \cong \mathbb{Z}$ is generated by $L_{t}$. For $t=0$ we get a cylinder and $\operatorname{Pic}\left(S_{t}\right) \cong \mathbb{Z} / 2$ is generated by $L_{0}$. As in the previous example, we see that $L^{2}$ is trivial on all infinitesimal neighborhoods of $S_{0}$.

\section{Existence of universal families}

9.1. Assume that we have a field $K$ that is finitely generated over its prime field and a local scheme $(x, X)$ of finite type over $K$. Write $P_{K}:=\mathbf{P i c}^{\text {loc-o }}(x, X)$.

As in Definition 8.4(1-4), there is a universal family

$$
\left(Y_{K} \stackrel{g_{K}}{\rightarrow} X_{K} \times P_{K} \rightarrow P_{K}, L_{Y_{K}}\right) .
$$

Everything in (9.1.1) can be defined over a finitely generated subring of $A \subset K$; thus there is an integral scheme $S$ of finite type over $\operatorname{Spec} \mathbb{Z}$ such that (9.1.1) is the generic fiber of a family

$$
\left(Y_{S} \stackrel{g_{S}}{\rightarrow} X_{S} \times P_{S} \rightarrow P_{S}, L_{Y_{S}}\right) .
$$

The statement and the proof of the next result closely follow [Art74]. I go through the details for two reasons. I always found [Art74] rather concise and, more importantly, not all the assumptions of [Art74] are satisfied in our case. There are two main differences. The automorphism groups of our objects are all infinite dimensional, but, as was already observed in [Bou78], this does not seem to cause any problems. A more difficult point is that the obstruction spaces are also infinite dimensional.

For the knowledgeable reader, Theorem 10.3 is the only part not contained in [Art74, Bou78]. THEOREM 9.2 (Openness of universality). Using the above notation, there is a dense open subset $T \subset S$ such that

$$
\left(Y_{T} \stackrel{g_{T}}{\rightarrow} X_{T} \times P_{T} \rightarrow P_{T}, L_{Y_{T}}\right)
$$

is everywhere universal (as in Definition 8.4).

Remark 9.3. One can imagine that

$$
P_{T} \quad \text { "=" } \mathbf{P i c}^{\mathrm{loc}-\circ}\left(\sigma_{T}, X_{T}\right),
$$

but we do not claim this. The main reason is that there are families of line bundles $L$ over $X_{S} \backslash \sigma(S)$ such that $L_{s} \in \mathbf{P i c}^{\text {loc-o }}\left(x_{s}, X_{s}\right)$ at the generic point but not at some special points. Consider for example the family

$$
X_{s}:=(x y=u v(u+v+s)) \subset \mathbb{C}^{4} \quad \text { and } \quad D_{s}:=(x=u=0)+(x=v=0) .
$$

For $s \neq 0$, we see that $D_{s} \sim(x=0)$ is trivial in $\operatorname{Pic}^{\text {loc }}\left(0, X_{s}\right)$. For $s=0$ we can use [Kol91, 2.2.7] to see that $\operatorname{Pic}^{\text {loc }}\left(0, X_{0}\right) \cong \mathbb{Z}^{2}$ with $(x=u=0)$ and $(x=v=0)$ as generators. Thus $D_{0}$ gives a nontorsion element in $\operatorname{Pic}^{\text {loc }}\left(0, X_{0}\right)=\operatorname{NS}^{\text {loc }}\left(0, X_{0}\right)$. I do not know whether such points $s \in S$ can be Zariski dense or not.

Thus $P_{T}$ should be viewed as an open neighborhood of the zero section in the (possibly nonexistent) $\mathbf{P i c}^{\text {loc-o}}\left(\sigma_{T}, X_{T}\right)$. 


\section{J. KOLLÁR}

Proof. By generic flatness we may assume that $P \rightarrow S$ is flat.

First, we prove that $\operatorname{Def}_{(s, S)}\left(\mathcal{O}_{Y_{s}}\right) \rightarrow \widehat{\mathcal{O}}_{\rho(s), P}$ is an isomorphism if and only if the map between the fibers over the points $\operatorname{Def}_{k(s)}\left(\mathcal{O}_{Y_{s}}\right) \rightarrow \widehat{\mathcal{O}}_{\rho(s), P_{s}}$ is an isomorphism. This is completely general and follows from Lemma 9.6.

Consider the tangent map

$$
t_{P_{K} / K}: T_{\rho(K), P_{K}} \rightarrow R^{1} j_{K} \mathcal{O}_{U_{K}}
$$

defined in (9.4.5). By assumption $t_{P_{K} / K}$ is an isomorphism. We prove in Proposition 9.5 that the tangent maps

$$
t_{P_{s} / s}: T_{\rho(s), P_{s}} \rightarrow R^{1} j_{s} \mathcal{O}_{U_{s}}
$$

are isomorphisms for all $s$ in a Zariski open subset of $S$.

If $P \rightarrow S$ is smooth, then a simple algebra result (Lemma 9.7) shows that $\operatorname{Def}_{k(s)}\left(\mathcal{O}_{Y_{s}}\right) \rightarrow$ $\hat{\mathcal{O}}_{\rho(s), P_{s}}$ is an isomorphism and we are done. If $\mathbf{P i c ^ { \text { loc-o} }}\left(x_{K}, X_{K}\right)$ is a smooth group scheme, then, possibly after shrinking $S$, we may assume that $P \rightarrow S$ is smooth. A group scheme over a field of characteristic 0 is always smooth, thus the proof of Theorem 9.2, and hence also the proofs of Theorems 1.4, 1.6 and 1.7, are complete if char $k=0$.

Otherwise we need a more detailed study of obstruction theory; this is started in $§ 9.8$.

9.4 (Tangent map). We continue with the notation of $\S 9.1$. Let $I_{S, P} \subset \mathcal{O}_{P}$ be the ideal sheaf of $\rho(S) \subset P$. We identify $S$ with $\rho(S)$ and set $R:=\operatorname{Spec}_{P} \mathcal{O}_{P} / I_{S, P}^{2}$. The ideal sheaf of $S \subset R$ is denoted by $I_{S}$. These data are encoded in a diagram

$$
\begin{aligned}
& Y_{S} \hookrightarrow Y_{R} \\
& \downarrow \quad g \downarrow \uparrow \sigma \quad \text { where } g \text { is flat, } \\
& S \hookrightarrow R \quad \sigma \text { is a section and } \\
& \| \quad \downarrow \quad I_{S}^{2}=0 \text {. } \\
& S=S
\end{aligned}
$$

Set $U_{R}:=Y_{R} \backslash \sigma(R)$ with natural injection $j: U_{R} \hookrightarrow Y_{R}$ and $U_{S}:=Y_{S} \backslash \sigma(S)$. Let $L_{R}$ be a line bundle on $U_{R}$ and $L_{S}:=\left.L_{R}\right|_{U_{S}}$. There is an exact sequence

$$
0 \rightarrow I_{S} \otimes_{S} L_{S} \rightarrow L_{R} \rightarrow L_{S} \rightarrow 0
$$

Pushing it forward by $j$, we get

$$
0 \rightarrow I_{S} \otimes_{S} j_{*} L_{S} \rightarrow j_{*} L_{R} \rightarrow j_{*} L_{S} \stackrel{\partial}{\rightarrow} I_{S} \otimes_{S} R^{1} j_{*} L_{S} .
$$

Now, assume that $L_{S} \cong \mathcal{O}_{U_{S}}$ and that $Y_{S} \rightarrow S$ has $S_{2}$ fibers. Then $j_{*} L_{S} \cong \mathcal{O}_{Y_{S}}$ and the exact sequence becomes

$$
0 \rightarrow I_{S} \otimes_{S} \mathcal{O}_{Y_{S}} \rightarrow j_{*} L_{R} \rightarrow \mathcal{O}_{Y_{S}} \stackrel{\partial}{\rightarrow} I_{S} \otimes_{S} R^{1} j_{*} \mathcal{O}_{Y_{S}}
$$

Here $\partial$ factors through $\mathcal{O}_{Y_{S}} \rightarrow \mathcal{O}_{Z} \cong \mathcal{O}_{S}$; thus, if $I_{S}$ is locally free over $\mathcal{O}_{S}$, then $\partial$ is equivalent to the tangent map

$$
t_{R / S}: \operatorname{Hom}_{S}\left(I_{S}, \mathcal{O}_{S}\right) \rightarrow R^{1} j_{*} \mathcal{O}_{Y_{S}}
$$

(Note that $\mathcal{H o m}_{S}\left(I_{S}, \mathcal{O}_{S}\right)$ is isomorphic to the relative tangent sheaf of $R / S$ restricted to $S \cong$ $\sigma(S)$.)

If we start with a set-up as in $\S 9.1$, then $R^{1} j_{*} \mathcal{O}_{Y_{S}} \cong R^{1} j_{*} \mathcal{O}_{X_{S}}$ and we get the following. 


\section{MAPS BetWeEN LOCAL PiCARD GRoups}

Proposition 9.5. Let $\left(Y_{S} \stackrel{g_{S}}{\rightarrow} X_{S} \times P_{S} \rightarrow P_{S}, S \stackrel{\rho_{S}}{\rightarrow} P_{S}, L_{Y_{S}}\right)$ be a deformation as in (9.1.2). Assume that $I_{S, P} / I_{S, P}^{2}$ is free over $S$ and that $R^{1} j_{*} \mathcal{O}_{X_{S}}$ is free and commutes with base change. Then the tangent map

$$
t_{P / S}: \operatorname{Hom}_{S}\left(I_{S, P}, \mathcal{O}_{S}\right) \rightarrow R^{1} j_{*} \mathcal{O}_{X_{S}}
$$

has constant rank over a dense open subset of $S$.

We have used the following commutative algebra lemmas.

Lemma 9.6. Let $(m, S)$ be a local ring and $\phi:\left(m_{1}, R_{1}\right) \rightarrow\left(m_{2}, R_{2}\right)$ a map of local $S$-algebras.

(i) If $R_{1}$ is complete and $\bar{\phi}: R_{1} / m R_{1} \rightarrow R_{2} / m R_{2}$ is surjective, then $\phi$ is surjective.

(ii) If $\phi$ is surjective, $\bar{\phi}$ is an isomorphism and $R_{2}$ is flat over $S$, then $\phi$ is an isomorphism.

Proof. For every $r \geqslant 1$, we have a commutative diagram

$$
\begin{array}{ccc}
\left(m^{r} / m^{r+1}\right) \otimes R_{1} / m R_{1} & \rightarrow & \left(m^{r} / m^{r+1}\right) \otimes R_{2} / m R_{2} \\
\downarrow & & \downarrow \\
m^{r} R_{1} / m^{r+1} R_{1} & \stackrel{a_{r}}{\rightarrow} & m^{r} R_{2} / m^{r+1} R_{2} .
\end{array}
$$

The vertical arrows are surjective; hence so is $a_{r}$. By induction on $r$ we obtain that the maps

$$
\bar{\phi}_{r}: R_{1} / m^{r+1} R_{1} \rightarrow R_{2} / m^{r+1} R_{2}
$$

are surjective. Since $R_{1}$ is complete, we can pass to the inverse limit to conclude that the maps

$$
R_{1}=\lim _{\longleftarrow} R_{1} / m^{r+1} R_{1} \rightarrow \underset{\lim }{\longleftarrow} R_{2} / m^{r+1} R_{2}
$$

are surjective. This factors through the injection $R_{2} \hookrightarrow \lim R_{2} / m^{r+1} R_{2}$; thus $\phi: R_{1} \rightarrow R_{2}$ is surjective.

For part (2), let $J$ be the kernel of $\phi$. We have an exact sequence

$$
0 \rightarrow J \rightarrow R_{1} \stackrel{\phi}{\rightarrow} R_{2} \rightarrow 0 .
$$

Since $R_{2}$ is flat over $S$, tensoring with $S / m_{S} S$ is also exact, thus we get

$$
0 \rightarrow J / m_{S} J \rightarrow R_{1} / m_{S} R_{1} \stackrel{\bar{\phi}}{\rightarrow} R_{2} / m_{S} R_{2} \rightarrow 0 .
$$

This implies $J / m_{S} J=0$; thus $J=0$ by the Nakayama lemma.

Lemma 9.7. Let $(R, m)$ be a complete local ring and $(S, n)$ a regular, local $(R, m)$-algebra. Then $R=S$ if and only if the natural maps $R / m \rightarrow S / n$ and $m / m^{2} \rightarrow n / n^{2}$ are isomorphisms.

Proof. If $R=S$, then clearly $R / m \rightarrow S / n$ and $m / m^{2} \rightarrow n / n^{2}$ are isomorphisms.

Conversely, assume that $R / m \rightarrow S / n$ and $m / m^{2} \rightarrow n / n^{2}$ are isomorphisms. By induction on $r$ we see that the natural maps $R / m^{r} \rightarrow S / n^{r}$ are surjective. Among local rings $\left(A, m_{A}\right)$ with fixed embedding dimension $\operatorname{dim}_{A / m} m_{A} / m_{A}^{2}$, the length of $S / n^{r}$ is the largest possible since $(S, n)$ is regular. Thus each $R / m^{r} \rightarrow S / n^{r}$ is an isomorphism. Since $(R, m)$ is complete, this implies that $R=S$.

9.8 (Obstruction map). Let $S$ be a base scheme and $R \rightarrow S$ a flat scheme with a section $\rho: S \rightarrow R$ with ideal sheaf $I_{S} \subset \mathcal{O}_{R}$. Let $T \subset R$ be a subscheme with ideal sheaf $I_{T}$. Assume $\rho(S) \subset T$ and $I_{S} I_{T}=0$. 


\section{J. KOLLÁR}

Let $g: Y_{R} \rightarrow R$ be a flat morphism with a section $\sigma: R \rightarrow Y_{R}$. By restriction, we get $Y_{S} \rightarrow S$ and $Y_{T} \rightarrow T$. These data are summarized in the following diagram:

$$
\begin{aligned}
& \begin{array}{cccccc}
Y_{S} & \hookrightarrow & Y_{T} & \hookrightarrow & Y_{R} \\
\downarrow & & \downarrow & & g \downarrow \uparrow \sigma
\end{array} \quad \text { where } g \text { is flat, } \\
& S \hookrightarrow T \hookrightarrow R \quad \sigma \text { is a section and } \\
& \| \quad \downarrow \quad \downarrow \quad I_{S} I_{T}=0 . \\
& S=S=S
\end{aligned}
$$

Finally, write $U_{R}:=Y_{R} \backslash \sigma(R), U_{T}:=Y_{T} \backslash \sigma(T)$ and $U_{S}:=Y_{S} \backslash \sigma(S)$, and let $L_{T}$ be a line bundle on $U_{T}$. We would like to understand when $L_{T}$ extends to a line bundle $L_{R}$ on $U_{R}$.

We have an exact sequence

$$
0 \rightarrow I_{T} \otimes_{S} \mathcal{O}_{U_{S}} \stackrel{m \mapsto 1+m}{\longrightarrow} \mathcal{O}_{U_{R}}^{*} \rightarrow \mathcal{O}_{U_{T}}^{*} \rightarrow 1
$$

This gives

$$
R^{1} j_{*} \mathcal{O}_{U_{R}}^{*} \rightarrow R^{1} j_{*} \mathcal{O}_{U_{T}}^{*} \stackrel{\partial}{\longrightarrow} I_{T} \otimes_{S} R^{2} j_{*} \mathcal{O}_{U_{S}}
$$

The line bundle $L_{T}$ corresponds to a section $\mathcal{O}_{S} \rightarrow R^{1} j_{*} \mathcal{O}_{U_{T}}^{*}$; composing with $\partial$ gives

$$
\left[L_{T}\right]: \mathcal{O}_{S} \rightarrow I_{T} \otimes_{S} R^{2} j_{*} \mathcal{O}_{U_{S}} .
$$

Thus $L_{T}$ extends to a line bundle $L_{R}$ if and only if $\left[L_{T}\right]=0$.

If $I_{T} / I_{S} I_{T}$ is free over $S$, then $\left[L_{T}\right]$ is equivalent to a map, called the obstruction,

$$
\operatorname{obs}\left(L_{T}, R\right): \operatorname{Hom}_{S}\left(I_{T}, \mathcal{O}_{S}\right) \rightarrow R^{2} j_{*} \mathcal{O}_{Y_{S}},
$$

and $L_{T}$ extends to a line bundle $L_{R}$ if and only if $\operatorname{obs}\left(L_{T}, R\right)=0$.

For us the following two consequences are especially important.

Claim 9.8.6. Assume that $S$ is integral, that $I_{T} / I_{S} I_{T}$ is free over $S$ and that $R^{2} j_{*} \mathcal{O}_{X_{S}}$ is free and commutes with base change over $S$.

(a) The obstruction map obs $\left(L_{T}, R\right)$ has constant rank and commutes with base change over a dense open subset of $S$.

(b) If obs $\left(L_{T}, R\right)$ is injective, then $L_{T}$ cannot be extended over any subscheme $T \subsetneq T^{\prime} \subset R$.

9.9 (End of the proof of Theorem 9.2). We return to the setting of (9.1.1) and, in addition, we choose a scheme $W_{K} \supset P_{K}$ such that $W_{K}$ is smooth at the identity and has the same tangent space as $P_{K}$. (Thus $W_{K}=P_{K}$ if $P_{K}$ is smooth.)

As before, everything is defined over a finitely generated subring $A \subset K$; thus there is an integral scheme $S$ of finite type over Spec $\mathbb{Z}$ such that (9.1.1) is the generic fiber of a family

$$
\left(Y_{S} \stackrel{g_{S}}{\rightarrow} X_{S} \times P_{S} \rightarrow P_{S}, W_{S} \supset P_{S}, L_{Y_{S}}\right) .
$$

In order to apply (9.8.6.a), denote by $\widehat{\mathcal{O}}_{S, W}$ the completion of the structure sheaf of $W_{S}$ along the section $\rho(S)$, by $\hat{I}_{S} \subset \widehat{\mathcal{O}}_{S, W}$ the ideal sheaf of $\rho(S)$ and by $\hat{I}_{P} \subset \widehat{\mathcal{O}}_{S, W}$ the ideal sheaf of $P_{S}$. Finally, set $T:=\operatorname{Spec}_{S} \widehat{\mathcal{O}}_{S, W} / \hat{I}_{P}$ and $R:=\operatorname{Spec}_{S} \widehat{\mathcal{O}}_{S, W} / \hat{I}_{S} \hat{I}_{P}$.

Shrinking $S$ if necessary, the following conditions can be satisfied:

(2.a) The schemes $T, R$ are flat over $S$ and $I_{T} / I_{S} I_{T}$ is free over $S$.

(2.b) The tangent map $t_{T / S}: \mathcal{H o m}_{S}\left(I_{S}, \mathcal{O}_{S}\right) \rightarrow R^{1} j_{*} \mathcal{O}_{X_{S}}$ is an isomorphism and commutes with base change. (This follows from Proposition 9.5.) 


\section{MAPS BetWeEN LOCAL PiCARD GRoups}

(2.c) The sheaf $R^{2} j_{*} \mathcal{O}_{Y_{S}}$ is free and commutes with base change. (This follows by applying Theorem 10.3 to $Y_{S} \rightarrow P_{S}$ and the sheaf $F=\mathcal{O}_{Y_{S}}$.)

Thus, by Claim 9.8.6, the line bundle $\left.L_{S}\right|_{T}$ does not extended over any subscheme $T \subsetneq T^{\prime} \subset R$. Therefore $P_{S} \rightarrow S$ is universal along the zero section $\rho: S \rightarrow P_{S}$. Since $P_{S} \rightarrow S$ is a group scheme, this implies that it is everywhere universal.

\section{Auxiliary results}

We discuss two theorems, Theorem 10.3 and Theorem 10.5, that are needed to complete our arguments. The preprint version of this paper contained proofs of the special cases that were used in our applications, but in both cases we now have much more general versions available.

The first topic is the generic freeness of higher direct images. A coherent sheaf over a reduced scheme is free over a dense open set, but there are quasi-coherent sheaves that are not free over any dense open subset. Even worse, a nonzero section may vanish at every closed point. The following two examples show what can happen.

Example 10.1. Let $M \subset \mathbb{Q}$ be the $\mathbb{Z}$-submodule consisting of all $m / n$ such that $n$ has no multiple prime factors. Let $\tilde{M}$ be the corresponding quasi-coherent sheaf over Spec $\mathbb{Z}$. The localization of $\tilde{M}$ at any prime ideal is free of $\operatorname{rank} 1$, but $\tilde{M}$ is not free. In fact, every global section of it vanishes at all but finitely many points of $\operatorname{Spec} \mathbb{Z}$.

Example 10.2. Let $E$ be an elliptic curve and $L$ the Poincaré bundle on $E \times E$. Set $X:=$ $\operatorname{Spec}_{E \times E} \sum_{i \geqslant 0} L^{i}$, and let $g: X \rightarrow E \times E \rightarrow E$ be a projection. Note that $g$ is smooth and has fiber dimension 2 , but it is neither affine nor proper.

If $e \in E$ is not a torsion point, then $H^{0}\left(X_{e}, \mathcal{O}_{X_{e}}\right)=H^{1}\left(X_{e}, \mathcal{O}_{X_{e}}\right)=0$. If $e \in E$ is a torsion point of order exactly $m$, then there are natural identifications

$$
H^{0}\left(X_{e}, \mathcal{O}_{X_{e}}\right) \cong H^{1}\left(X_{e}, \mathcal{O}_{X_{e}}\right) \cong \sum_{i \in m \mathbb{N}} k(e) .
$$

Thus we see that $g_{*} \mathcal{O}_{X}=0$ is free, but it does not commute with base change over any open set. By contrast, $R^{1} g_{*} \mathcal{O}_{X}$ is a sum of skyscraper sheaves of infinite rank supported at the torsion points. Thus it is not generically free, but it does commute with base change.

Theorem 10.3 [Smi16]. Let $f: X \rightarrow S$ be a finite-type morphism of Noetherian schemes with $S$ integral. Let $Z \subset X$ be a subscheme such that $\left.f\right|_{Z}$ is finite. Set $U:=X \backslash Z$, and let $j: U \hookrightarrow X$ be the natural injection. Let $F$ be a coherent sheaf on $U$ that is flat over $S$. Then there is a dense, open subscheme $S^{0} \subset S$ such that, for every $r \geqslant 1$,

(i) $f_{*} \circ R^{r} j_{*} F$ is free over $S^{0}$ and

(ii) the formation of $f_{*} \circ R^{r} j_{*} F$ commutes with base changes $W \rightarrow S^{0} \hookrightarrow S$.

10.4 (Proof of Theorem 1.9). We assume $\operatorname{depth}_{x} X \geqslant 3$; hence, $\mathbf{P i c}^{\text {loc }}(x, X)$ is 0 dimensional. A 0 -dimensional linear algebraic group is finite. This shows (1.9.2-3). Thus the only new claim is that $r_{D}^{X}: \mathbf{P i c}^{\text {loc }}(x, X) \longrightarrow \mathbf{P i c}^{\text {loc }}(x, D)$ is injective (1.9.1).

We already noted in $\S 2.5$ that every torsion element in the kernel of $r_{D}^{X}$ has $p$-power order, where $p$ is the residue characteristic. The proof relied on the observation that for $p \nmid m$, a torsion element of order $m$ in $\operatorname{Pic}^{\text {loc }}(x, X)$ corresponds to a degree $m$ étale cover of $X \backslash\{x\}$ and then used [Gro68, XIII.2.1].

The following result completes the proof of Theorem 1.9. 


\section{J. KOLLÁR}

Theorem 10.5 [dJ15, Proposition 3]. Let $(x, X)$ be a local, Noetherian scheme and $X \in D \subset X$ a Cartier divisor. Let $x \in Z \subset D$ be a closed subscheme such that $\operatorname{depth}_{Z} D \geqslant 2$. Then

$$
\operatorname{ker}\left[r_{D}^{X}: \operatorname{Pic}(X \backslash Z) \rightarrow \operatorname{Pic}(D \backslash Z)\right] \quad \text { is torsion free. }
$$

\section{Numerical criteria for relative Cartier divisors}

Definition 11.1. Let $T$ be a regular 1-dimensional scheme and $f: X \rightarrow T$ a flat morphism with $S_{2}$ fibers. A generically flat family of divisors $D$ on $X$ is given by

(i) an open set $U \subset X$ such that $\operatorname{codim}_{X_{t}}\left(X_{t} \backslash U\right) \geqslant 2$ for every $t \in T$ and

(ii) a relative Cartier divisor $D_{U}$ on $U$, that is, a Cartier divisor whose restriction to every fiber is a Cartier divisor.

For each $t \in T$, set $U_{t}:=X_{t} \cap U$. Then $\left.D_{U}\right|_{U_{t}}$ extends uniquely to a divisor on $X_{t}$; we denote it by $D_{t}$ and call it the restriction of $D$ to $X_{t}$.

If $|H|$ is a base-point-free linear system on $X$, then the restriction of $D$ to a general $H \in|H|$ is again a generically flat family of divisors.

We say that $D$ is a fiberwise Cartier family of divisors if each $D_{t}$ is a Cartier divisor. It is easy to see that

$$
r_{t}:\left.\mathcal{O}_{X}(D)\right|_{X_{t}}=\left.\left(j_{*} \mathcal{O}_{U}\left(D_{U}\right)\right)\right|_{X_{t}} \rightarrow\left(j_{t}\right)_{*}\left(\mathcal{O}_{U_{t}}\left(D_{U_{t}}\right)\right)=\mathcal{O}_{X_{t}}\left(D_{t}\right)
$$

is an isomorphism for general $t \in T$; thus $D$ is Cartier except possibly along finitely many closed fibers.

Our main interest is to find conditions that guarantee that a fiberwise Cartier family of divisors is everywhere Cartier.

We say that $D$ is a fiberwise ample family of divisors if each $D_{t}$ is an ample Cartier divisor.

We concentrate on the cases when $T$ is local with closed point 0 and generic point $g$. The following is a direct consequence of the Nakayama lemma.

Lemma 11.2. We take the notation and assumptions of Definition 11.1. Then $D$ is Cartier if and only if $D_{0}$ is Cartier and $r_{0}:\left.\mathcal{O}_{X}(D)\right|_{X_{0}} \rightarrow \mathcal{O}_{X_{0}}\left(D_{0}\right)$ is an isomorphism.

11.3 (Numerical inequalities). We take the notation and assumptions of Definition 11.1. Assume in addition that $f$ is proper and has relative dimension $n$. By semicontinuity

$$
H^{0}\left(X_{0}, \mathcal{O}_{X_{0}}\left(D_{0}\right)\right) \geqslant H^{0}\left(X_{0},\left.\mathcal{O}_{X}(D)\right|_{X_{0}}\right) \geqslant H^{0}\left(X_{g}, \mathcal{O}_{X_{g}}\left(D_{g}\right)\right) \text {. }
$$

In particular, if $D_{g}$ is ample on $X_{g}$ and $D_{0}$ is Cartier and ample on $X_{0}$, then, applying (11.3.1) to multiples of $D$, we conclude that

$$
\left(D_{0}^{n}\right) \geqslant\left(D_{g}^{n}\right) \text {. }
$$

One can be more precise if $\operatorname{dim} Z_{0}=0$. Then coker $r_{0}$ is artinian, thus

$$
\chi\left(X_{0}, \mathcal{O}_{X_{0}}\left(D_{0}\right)\right)=\chi\left(X_{0},\left.\mathcal{O}_{X}(D)\right|_{X_{0}}\right)+\operatorname{length}\left(\operatorname{coker} r_{0}\right) .
$$

Since $\mathcal{O}_{X}(D)$ is flat over $T$,

$$
\chi\left(X_{0},\left.\mathcal{O}_{X}(D)\right|_{X_{0}}\right)=\chi\left(X_{g},\left.\mathcal{O}_{X}(D)\right|_{X_{g}}\right)=\chi\left(X_{g}, \mathcal{O}_{X_{g}}\left(D_{g}\right)\right) .
$$

Combining these, we see that

$$
\chi\left(X_{0}, \mathcal{O}_{X_{0}}\left(D_{0}\right)\right)=\chi\left(X_{g}, \mathcal{O}_{X_{g}}\left(D_{g}\right)\right)+\text { length }\left(\operatorname{coker} r_{0}\right) .
$$




\section{MAPS BeTWEen LOCAL PiCARD GROUPS}

Together with Lemma 11.2, this proves the following.

Corollary 11.4. We take the notation and assumptions of Definition 11.1. Assume in addition that $f$ is proper and $\operatorname{dim} Z_{0}=0$. Then $D$ is Cartier if and only if $D_{0}$ is Cartier and

$$
\chi\left(X_{0}, \mathcal{O}_{X_{0}}\left(D_{0}\right)\right)=\chi\left(X_{g}, \mathcal{O}_{X_{g}}\left(D_{g}\right)\right) .
$$

Combining Theorems 1.9 and 10.5, we have the following.

Theorem 11.5. Let $T$ be an irreducible, regular, 1-dimensional scheme and $f: X \rightarrow T$ a flat morphism that is essentially of finite type and has $S_{2}$ fibers.

Let $D$ be a generically flat family of fiberwise Cartier divisors on $X$. Assume that there is a closed subscheme $W \subset X$ such that $\operatorname{codim}_{X_{t}}\left(X_{t} \cap W\right) \geqslant 3$ for every $t \in T$ and that $D$ is Cartier on $U:=X \backslash W$. Then $D$ is a Cartier divisor on $X$.

It remains to understand what happens when the putative non-Cartier locus has codimension 2. For projective morphisms we have the following numerical criteria.

THEOREM 11.6. Let $T$ be an irreducible, regular, 1-dimensional scheme and $f: X \rightarrow T$ a flat, projective morphism of relative dimension $n$ with $S_{2}$ fibers.

Let $D$ be a generically flat family of fiberwise Cartier divisors on $X$ and $H$ an $f$-ample Cartier divisor on $X$. The following are equivalent:

(i) The family $D$ is a Cartier divisor on $X$.

(ii) The intersection number $\left(D_{t}^{2} \cdot H_{t}^{n-2}\right)$ is independent of $t \in T$.

If $D$ is fiberwise ample, then these are further equivalent to the following:

(iii) The self-intersection number $\left(D_{t}^{n}\right)$ is independent of $t \in T$.

Proof. If $D$ is Cartier, then all the intersection numbers $\left(D_{t}^{i} \cdot H_{t}^{n-i}\right)$ are independent of $t \in T$. Thus conditions (1) implies conditions (2) and (3).

To see the converse, we may assume that $T$ is local with closed point $0 \in T$ and generic point $g \in T$. Let $Z_{0} \subset X$ be the smallest closed subset such that $D$ is Cartier on $X \backslash Z_{0}$. Note that $Z_{0} \subset X_{0}$ since $D$ is Cartier on $X_{g}$. We can choose $U:=X \backslash Z_{0}$ as the open set in Definition 11.1.

First, assume that $n=2$, thus conditions (2) and (3) coincide. For each $t \in T$, the Euler characteristic is a quadratic polynomial

$$
\chi\left(X_{t}, \mathcal{O}_{X_{t}}\left(m D_{t}\right)\right)=a_{t} m^{2}+b_{t} m+c_{t},
$$

and we know from the Riemann-Roch theorem that $a_{t}=\frac{1}{2}\left(D_{t}^{2}\right)$ and $c_{t}=\chi\left(X_{t}, \mathcal{O}_{X_{t}}\right)$. Furthermore, (11.3.4) implies that

$$
a_{0} m^{2}+b_{0} m+c_{0} \geqslant a_{g} m^{2}+b_{g} m+c_{g} \quad \text { for every } m \in \mathbb{Z} .
$$

For $m \gg 1$, the quadratic terms dominate, which gives

$$
\left(D_{0}^{2}\right)=2 a_{0} \geqslant 2 a_{g}=\left(D_{g}^{2}\right) .
$$

Now, assume $\left(D_{0}^{2}\right)=\left(D_{g}^{2}\right)$. Then $a_{0}=a_{g}$, thus (11.6.5) implies that

$$
b_{0} m+c_{0} \geqslant b_{g} m+c_{g} \quad \text { for every } m \in \mathbb{Z} .
$$

For $m \gg 1$, this implies that $b_{0} \geqslant b_{g}$, and for $m \ll-1$ it implies that $-b_{0} \geqslant-b_{g}$. Thus $b_{0}=b_{g}$ and $c_{0}=\chi\left(X_{0}, \mathcal{O}_{X_{0}}\right)=\chi\left(X_{g}, \mathcal{O}_{X_{g}}\right)=c_{g}$ also holds since $f$ is flat. Therefore, we have equality 


\section{J. Kollár}

in (11.6.4). Using Corollary 11.4, we see that

$$
r_{0}:\left.j_{*} \mathcal{O}_{U}\left(D_{U}\right)\right|_{X_{0}} \rightarrow\left(j_{0}\right)_{*} \mathcal{O}_{U_{0}}\left(D_{0}\right)
$$

is an isomorphism and hence $\mathcal{O}_{X}(D)=j_{*} \mathcal{O}_{U}\left(D_{U}\right)$ is locally free.

In order to prove that condition (2) implies condition (1) for $n \geqslant 3$, we use induction on $n$. A suitable multiple $|m H|$ provides an embedding $X \subset \mathbb{P}_{T}^{N}$; let $X^{\prime} \subset X$ be a general hyperplane section. Then $f^{\prime}:=\left.f\right|_{X^{\prime}}: X^{\prime} \rightarrow T$ is a flat, projective morphism of relative dimension $n-1$ and $Z^{\prime}:=Z \cap X^{\prime}$ is a closed subscheme such that $\operatorname{codim}_{X_{t}^{\prime}}\left(X_{t}^{\prime} \cap Z^{\prime}\right) \geqslant 2$ for every $t \in T$.

Furthermore, $D^{\prime}:=\left.D\right|_{X^{\prime}}$ is a divisor, Cartier over $U^{\prime}:=X^{\prime} \backslash Z^{\prime}$, whose support does not contain any irreducible component of a fiber of $f^{\prime}$, and $\left.D^{\prime}\right|_{X_{t}^{\prime}}$ is Cartier for every $t$. Finally, $\left(\left(D_{t}^{\prime}\right)^{2} \cdot H_{t}^{n-3}\right)=m\left(D_{t}^{2} \cdot H_{t}^{n-2}\right)$ is independent of $t \in T$.

Since $f$ has $S_{2}$ fibers, a hyperplane section of it usually has only $S_{1}$ fibers. However, by [Gro60, IV.12.1.6], a general hyperplane section again has $S_{2}$ fibers. Thus, by induction, $D^{\prime}=\left.D\right|_{X^{\prime}}$ is a Cartier divisor. As we noted in Definition 11.1, since $X^{\prime}$ is general, this implies that $D$ is Cartier along $X^{\prime}$. Hence, there is a closed subscheme $W \subset X$ such that $W \cap X_{t}$ is 0 dimensional for every $t \in T$ and $D$ is Cartier on $X \backslash W$. Thus $D$ is Cartier by Theorem 11.5.

Finally, assume that $D$ is fiberwise ample. Choose $m>0$ such that $m D_{0}-H_{0}$ and $m D_{t}-H_{t}$ are ample. Thus $B:=m D-H$ is also fiberwise ample and

$$
m^{n}\left(D_{t}^{n}\right)=\sum_{i=0}^{n}\left(B_{t}^{i} \cdot H_{t}^{n-i}\right) .
$$

We can apply (11.3.2) to the restriction of $B$ to an intersection of $n-i$ general members of $H$ to obtain that $\left(B_{0}^{i} \cdot H_{0}^{n-i}\right) \geqslant\left(B_{g}^{i} \cdot H_{g}^{n-i}\right)$ for every $i$. However, $\left(D_{0}^{n}\right)=\left(D_{g}^{n}\right)$ by condition (3), thus $\left(B_{0}^{i} \cdot H_{0}^{n-i}\right)=\left(B_{g}^{i} \cdot H_{g}^{n-i}\right)$ for every $i$. We can use this for $i=2$ and the already established fact that condition (2) implies condition (1) to conclude that $B$ is Cartier. Thus $m D=B+H$ is also Cartier. By the same argument $(m+1) D$ is also Cartier; this implies that $D$ is Cartier.

\section{Open problems}

Finite generation of $\operatorname{NS}^{\text {loc }}(x, X)$

The main unsolved problem is the finite generation of $\mathrm{NS}^{\text {loc }}(x, X)$. This is known if $X$ is normal or if the characteristic is 0 and $\operatorname{dim} X \geqslant 3$. In positive characteristic, our methods do not seem to distinguish a unipotent subgroup of $\mathbf{P i c}{ }^{\text {loc-o }}$ from a discrete $p$-group.

For surfaces over $\mathbb{C}$, even stronger results should hold; see Conjecture 12.4.

\section{Local Picard group of excellent schemes}

While our theorems settle only the geometric cases, I see no reason why they should not hold in general.

Conjecture 12.1. Let $X$ be an excellent scheme that is $S_{2}$ and has pure dimension at least 3 . Let $x \in X$ be a closed point and $\bar{X} \rightarrow X$ the normalization. Then $\operatorname{ker}\left[\pi^{*}: \operatorname{Pic}^{\text {loc }}(x, X) \rightarrow\right.$ $\left.\operatorname{Pic}^{\text {loc }}(\bar{x}, \bar{X})\right]$ is a linear algebraic group.

Conjecture 12.2. Let $X$ be an excellent scheme that is $S_{2}$ and has pure dimension at least 4 . Let $x \in X$ be a closed point and $x \in D \subset X$ an effective Cartier divisor. Then $\operatorname{ker}\left[r_{D}^{X}: \mathbf{P i c}^{\operatorname{loc}}(x, X) \rightarrow\right.$ $\left.\mathbf{P i c}^{\text {loc }}(x, D)\right]$ is a unipotent algebraic group. 


\section{MAPS BetWeEN LOCAL PiCARD GRoups}

Note that not even the existence of $\mathbf{P i c}{ }^{\text {loc }}(x, X)$ is known in the above generality, but algebraic equivalence can be defined as in Definition 3.3.

\section{Local Picard group of surfaces}

The construction of [Bou78] does not apply to surfaces; in fact, the functorial approach always gives the "wrong" answer. For normal surface singularities over $\mathbb{C}$, Mumford [Mum61] constructed a finite-dimensional local Picard group Pic ${ }^{\text {loc }}(s, S)$. Example 4.5 (in particular, (4.5.3) and (4.5.4)) shows that in many nonnormal cases there is no finite-dimensional local Picard group.

Conjecture 12.3. Let $(s, S)$ be an $S_{2}$ surface. Then there is a finite-dimensional local Picard group $\mathbf{P i c}^{\text {loc }}(s, S)$ if and only if $S$ is seminormal.

Conjecture 12.4. Let $(x, X)$ be a local $\mathbb{C}$-scheme of finite type that is $S_{2}$ and has pure dimension 2. Then taking the first Chern class gives an exact sequence

$$
0 \rightarrow \mathbf{P i c}^{\operatorname{loc}-\circ}(x, X) \rightarrow \mathbf{P i c}^{\operatorname{loc}}(x, X) \stackrel{c_{1}}{\rightarrow} H^{2}(\operatorname{link}(x, X), \mathbb{Z}) .
$$

The normal case is discussed in [Mum61]. It would be nice to have something similar in positive characteristic, but I do not know what should replace $H^{2}(\operatorname{link}(x, X), \mathbb{Z})$. See also Example 4.8 .

In connection with Example 4.4, one can ask the following.

Question 12.5. Let $\left(0, S^{\text {an }}\right)$ be a normal, analytic surface singularity over $\mathbb{C}$ such that $\mathbf{P i c}^{\operatorname{loc}-\circ}(0, S)$ is proper. Is there an algebraic model $(0, S)$ such that $\operatorname{Pic}^{\operatorname{loc}}(0, S)=\operatorname{Pic}^{\text {loc }}\left(0, S^{\text {an }}\right)$ ?

\section{Second cohomology of links}

One can refine the topological approach of Section 5 using the mixed Hodge structures on the cohomology groups of the $\operatorname{links} H^{2}(\operatorname{link}(x, X), \mathbb{C})$ and $H^{2}(\operatorname{link}(\bar{x}, \bar{X}), \mathbb{C})$ [PS08, Section 6.2]. Since the Chern class of a line bundle has pure Hodge type $(1,1)$, the following would imply most of Conjecture 12.1 for analytic spaces.

Conjecture 12.6. Let $X$ be a complex analytic space that is $S_{2}$ and has pure dimension at least 3 . Let $x \in X$ be a point. Then the pull-back map

$$
\pi^{*}: H^{2}(\operatorname{link}(x, X), \mathbb{C}) \rightarrow H^{2}(\operatorname{link}(\bar{x}, \bar{X}), \mathbb{C})
$$

is injective on the weight 2 graded piece of the mixed Hodge structure.

\section{Obstruction theory for Pic ${ }^{\text {loc }}$}

The usual obstruction space for $\mathbf{P i c}^{\text {loc }}(x, X)$ is $H_{x}^{3}\left(X, \mathcal{O}_{X}\right)$. This space is infinite dimensional if $\operatorname{dim} X=3$ or if $\operatorname{dim} X \geqslant 4$ and $X \backslash\{x\}$ is not $S_{3}$.

Already, Artin [Art74] observed that an obstruction theory is an external construct imposed on a functor and that there could be different "natural" obstruction theories that work for the same functor. Theorem 10.3 suggests that one should be able to develop a finite-dimensional obstruction theory for Pic ${ }^{\text {loc }}$. I do not know how to formulate such a theory.

\section{ACKNOWLEDGEMENTS}

I thank B. Bhatt, J. de Jong and K. Smith for answering my questions and suggesting many 


\section{J. KOLLÁR}

improvements.

\section{REFERENCES}

Art69 M. Artin, Algebraic approximation of structures over complete local rings, Publ. Math. Inst. Hautes Études Sci. 36 (1969), no. 1, 23-58; http://dx.doi.org/10.1007/BF02684596.

Art74 Versal deformations and algebraic stacks, Invent. Math. 27 (1974), no. 3, 165-189; http://dx.doi.org/10.1007/BF01390174.

Bar55 I. Barsotti, Un teorema di struttura per le varietà gruppali, Atti Accad. Naz. Lincei. Rend. Cl. Sci. Fis. Mat. Nat. (8) 18 (1955), 43-50.

BdJ14 B. Bhatt and A. J. de Jong, Lefschetz for local Picard groups, Ann. Sci. Éc. Norm. Supér. (4) 47 (2014), no. 4, 833-849.

BLR90 S. Bosch, W. Lütkebohmert and M. Raynaud, Néron models, Ergeb. Math. Grenzgeb. (3), vol. 21 (Springer-Verlag, Berlin, 1990); http://dx.doi .org/10.1007/978-3-642-51438-8.

Bor91 A. Borel, Linear algebraic groups, 2nd ed., Grad. Texts in Math., vol. 126 (Springer-Verlag, New York, 1991); http://dx.doi.org/10.1007/978-1-4612-0941-6.

Bou78 J.F. Boutot, Schéma de Picard local, Lecture Notes in Math., vol. 632 (Springer, Berlin, 1978); http://dx.doi.org/10.1007/BFb0068424.

BSU13 M. Brion, P. Samuel and V. Uma, Lectures on the structure of algebraic groups and geometric applications, CMI Lect. Series in Math., vol. 1 (Hindustan Book Agency, New Delhi, Chennai Math. Inst., Chennai, 2013).

Gro60 A. Grothendieck and J. Dieudonné, Éléments de géométrie algébrique, Publ. Math. Inst. Hautes Études Sci. 4 (Chapter 0, 1-7, and I, 1-10), 8 (II, 1-8), 11 (Chapter 0, 8-13, and III, 1-5), $\mathbf{1 7}$ (III, 6-7), 20 (Chapter 0, 14-23, and IV , 1), 24 (IV, 2-7), 28 (IV, 8-15), and 32 (IV, 16-21), 1960-1967; available at http://www.numdam.org.

Gro68 A. Grothendieck, Cohomologie locale des faisceaux cohérents et théorèmes de Lefschetz locaux et globaux (Séminaire de Géométrie Algébrique du Bois-Marie 1962 (SGA 2)), Adv. Stud. Pure Math., vol. 2 (North-Holland Publishing Co., Amsterdam; Masson \& Cie, Éditeur, Paris, 1968).

Har62 R. Hartshorne, Complete intersections and connectedness, Amer. J. Math. 84 (1962), 497-508; http://dx.doi.org/10.2307/2372986.

dJ96 A. J. de Jong, Smoothness, semi-stability and alterations, Publ. Math. Inst. Hautes Études Sci. 83 (1996), 51-93; http://dx.doi.org/10.1007/BF02698644.

dJ15 Local Picard groups, lecture at the AMS Summer Institute in Algebraic Geometry, Salt Lake City, UT, 30 July 2015; recording available online at http: //www. claymath.org/downloads/utah/SFEBB\%201110\%20-\%20Week\%203/Local\%20Picard\% 20 groups $\% 20-\% 20$ Johan $\% 20$ de $\% 20$ Jong.mp4.

Kle66 S. L. Kleiman, Toward a numerical theory of ampleness, Ann. of Math. 84 (1966), 293-344; http://dx.doi.org/10.2307/1970447.

KM98 J. Kollár and S. Mori, Birational geometry of algebraic varieties, Cambridge Tracts in Math., vol. 134 (Cambridge Univ. Press, Cambridge, 1998); http://dx.doi.org/10.1017/ CB09780511662560.

Kol91 J. Kollár, Flips, flops, minimal models, etc, Surveys in Differential Geometry (Cambridge, MA, 1990) (Lehigh University, Bethlehem, PA, 1991), 113-199.

Kol96 , Rational curves on algebraic varieties, Ergeb. Math. Grenzgeb. (3) Folge A, vol. 32 (Springer-Verlag, Berlin, 1996); http://dx.doi .org/10.1007/978-3-662-03276-3.

Kol97 , Quotient spaces modulo algebraic groups, Ann. of Math. 145 (1997), no. 1, 33-79; http: //dx.doi.org/10.2307/2951823.

Kol11_, Sources of log canonical centers, 2011, arXiv:1107.2863. 


\section{MAPS BetWeEN LOCAL PiCARD GRoups}

Kol12 , Quotients by finite equivalence relations, Current Developments in Algebraic Geometry, Math. Sci. Res. Inst. Publ., vol. 59 (Cambridge Univ. Press, Cambridge, MA, 2012), 227-256.

Kol13a _ Grothendieck-Lefschetz type theorems for the local Picard group, J. Ramanujan Math. Soc. 28A (2013), 267-285.

Kol13b___ Singularities of the minimal model program, Cambridge Tracts in Math., vol. 200 (Cambridge Univ. Press, Cambridge, 2013); http://dx.doi.org/10.1017/CB09781139547895.

Mum61 D. Mumford, The topology of normal singularities of an algebraic surface and a criterion for simplicity, Publ. Math. Inst. Hautes Études Sci. 9 (1961), 5-22; http://dx.doi.org/10.1007/ BF02698717.

PS94 A.J. Parameswaran and V. Srinivas, A variant of the Noether-Lefschetz theorem: some new examples of unique factorisation domains, J. Algebraic Geom. 3 (1994), no. 1, 81-115.

PS08 C.A.M. Peters and J.H.M. Steenbrink, Mixed Hodge structures, Ergeb. Math. Grenzgeb. (3) Folge A, vol.52 (Springer-Verlag, Berlin, 2008); http://dx.doi.org/10.1007/ 978-3-540-77017-6.

Sch68 M. Schlessinger, Functors of Artin rings, Trans. Amer. Math. Soc. 130 (1968), 208-222; http: //dx.doi.org/10.1090/S0002-9947-1968-0217093-3.

Siu69 Y.-T. Siu, Extending coherent analytic sheaves, Ann. of Math. 90 (1969), 108-143; http://dx. doi.org/10.2307/1970684.

Smi16 K. E. Smith, Local cohomology and base change, 2015, arXiv:1607.00062.

János Kollár kollar@math.princeton.edu

Department of Mathematics, Fine Hall, Washington Road Princeton University,

Princeton, NJ 08544-1000, USA 